\title{
A Holistic and Probabilistic Approach to the Ground-based and Spaceborne Data of HAT-P-19 System
}

\author{
Özgür Baştürk ${ }^{1}{ }^{\star}$ S. Yalçınkaya ${ }^{1}$, E. M. Esmer ${ }^{1}$, T. Tanrıverdi ${ }^{2}$, L. Mancini ${ }^{3,4,5}$ \\ T. Daylan ${ }^{6,7}$, J. Southworth ${ }^{8}$, B. Keten ${ }^{1}$ \\ ${ }^{1}$ Ankara University, Faculty of Science, Astronomy \&S Space Sciences Department, Tandogan, TR-06100, Ankara, Turkey \\ ${ }^{2}$ Niğde Ömer Halisdemir University, Faculty of Arts and Sciences, Department of Physics, TR-51240, Niğde, Turkey \\ ${ }^{3}$ Department of Physics, University of Rome "Tor Vergata", Via della Ricerca Scientifica 1, I-00133, Rome, Italy \\ ${ }^{4}$ Max Planck Institute for Astronomy, Königstuhl 17, D-69117, Heidelberg, Germany \\ ${ }^{5}$ INAF - Osservatorio Astrofisico di Torino, via Osservatorio 20, I-10025, Pino Torinese, Italy \\ 6 Department of Physics and Kavli Institute for Astrophysics and Space Research, Massachusetts Institute of Technology, \\ 70 Vassar Street, Cambridge, MA 02139, USA \\ 7 Kavli Fellow \\ 8 Astrophysics Group, Keele University, Staffordshire ST5 5BG, UK
}

Accepted XXX. Received YYY; in original form ZZZ

\begin{abstract}
We update the main physical and orbital properties of the transiting hot Saturn planet HAT-P$19 \mathrm{~b}$, based on a global modelling of high-precision transit and occultation light curves, taken with ground-based and space telescopes, archive spectra and radial velocity measurements, brightness values from broadband photometry, and Gaia parallax. We collected 65 light curves by amateur and professional observers, measured mid-transit times, analyzed their differences from calculated transit timings based on reference ephemeris information, which we update as a result. We haven't found any periodicity in the residuals of a linear trend, which we attribute to the accumulation of uncertainties in the reference mid-transit time and the orbital period. We comment on the scenarios describing the formation and migration of this hot-Saturn type exoplanet with a bloated atmosphere yet a small core, although it is orbiting a metal-rich $([\mathrm{Fe} / \mathrm{H}]=0.24 \mathrm{dex})$ host star. Finally, we review the planetary mass-radius, the orbital period-radius and density, and the stellar metallicity-core mass diagrams, based on the parameters we derive for HAT-P-19 b and those of the other seventy transiting Saturn-mass planets from the NASA Exoplanet Archive.
\end{abstract}

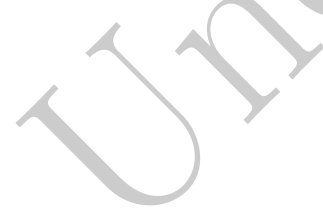

Key words: planets and satellites: individual: HAT-P-19 b - planetary systems methods: observational - techniques: photometric - techniques: spectroscopic - techniques : timing - stars: individual: HAT-P-19

\section{INTRODUCTION}

Transiting exoplanets are of interest for several reasons. First of all, many physical properties of the planet and the host star can be directly measured or derived based on a minimum set of assumptions and the established theory of stellar evolution. Furthermore, variations in their transit timings can be indicative of an unstable orbit or additional bodies gravitationally bound to the system. In addition, planet formation and migration mechanisms of close-in giant planets can be tested based on a sample space containing wellcharacterized planets that have been discovered frequently in transit surveys due to the biases of the technique. As of now, even atmospheric constituents and properties of a handful of such planets have been constrained. There is cur-

^ E-mail: obasturk@ankara.edu.tr rently a big effort to probe the atmospheres of more and more of these exoplanets, thanks to new highly performing instruments. This kind of investigations should become more accurate with the JWST and future extremely-largeclass telescopes. Therefore, it is very worthwhile to monitor these systems frequently, analyze their archival data and study them in more detail. From this perspective, the aim of this work is to refine the physical parameters of the planetary system HAT-P-19, mainly based on the analysis of $(i)$ new and precise photometric follow-up observations and (ii) high resolution spectra from the Keck/HIRES archive. We also investigate possible Transit Timing Variations (TTV) of the hot-Saturn HAT-P-19 b by analyzing our own observations, the light curves recorded by the Transiting Exoplanets Survey Satellite (TESS) (Ricker et al. 2015) and Spitzer telescopes, as well as other ground-based photometric observations of professional and amateur observers. 
HAT-P-19 b is a low-density $\left(\rho_{\mathrm{p}}=0.293 \mathrm{~g} \mathrm{~cm}^{-3}\right)$, Saturn-mass $\left(M_{\mathrm{p}}=0.95 \mathrm{M}_{\text {Saturn }}\right)$ exoplanet orbiting a metal-rich $([\mathrm{Fe} / \mathrm{H}]=+0.243 \mathrm{dex})$ star, on a $P_{\text {orb }}=4.01$ day orbit with an eccentricity consistent with zero $(<0.024$; $1 \sigma$ upper limit; Bonomo et al. 2017). Its host star is a typical K-type main-sequence star with $T_{\text {eff }} \sim 4970 \mathrm{~K}$, and log $g=4.567$. Most of the confirmed transiting planets with a mass roughly equal to that of Saturn have lower densities than that of the pure water, orbiting their host stars with diverse physical and orbital properties. Therefore, it is crucial to determine the parameters of such systems accurately and precisely to carry out a population analysis of the planets within this mass regime to understand their formation, orbital evolution, and the correlations between their parameters and that of their host stars.

High iron abundance in the host star's atmosphere, in contrast of the low density of its planet makes it a contradictory example for the correlation suggested to be existing between the core mass of the planet and the metallicity of the star in this mass regime (Hartman et al. 2011) which was inspired by a similar correlation between the radius of the planet and the metallicity of the host star for slightly more massive $\left(0.4-0.7 M_{\text {jup }}\right)$ planets (Enoch et al. 2011). This sub-group of gas giants with intermediate masses $\left(0.1 \mathrm{M}_{\text {jup }}<M_{\mathrm{p}}<0.4 M_{\text {jup }}\right)$, low densities $\left(\rho_{\mathrm{p}}<2.00\right.$ $\left.\mathrm{g} / \mathrm{cm}^{3}\right)$, and relatively small orbits $(a<1.0 \mathrm{au})$ are called hot-Saturns. Together with their larger cousins, called hotJupiters, they pose a strong challenge for core-accretion scenario, requiring a migration mechanism to drag the planets from where they formed to where they are/observed now. However, a relatively recent study has asserted insitu formation within core-accretion scenario as a potential mechanism for the formation of hot-Jupiters and Saturns (Bailey \& Batygin 2018). The observed radius anomaly in these hot and bloated Saturns can be explained considering the incident flux coming from their host stars (Lundkvist et al. 2016). However, the core mass of HATP-19 b, computed from theoretical models of Fortney et al. (2007), is still below the limits expected from the high metal content in such proximity of super-solar metallicity stars (Feltzing \& Gonzalez 2001), where they might be claimed to have formed. In the case of in-situ formation, kinetic heating (Guillot \& Showman 2002) might be the mechanism that can unravel the story behind the inflation of such planets orbiting metal-rich stars. HAT-P-19 b is an important member of this class of planets, whose cores are expected to be enriched in metal content, therefore, should have been larger if they form where they are observed. If they had formed outside the snow-line and have migrated inwards, then the reason behind the inflation of their atmosphere, as well as the type of their migration history, are the questions to answer.

HAT-P-19 b's extended radius $\left(1.064 R_{\text {jup }}\right)$ for its mass $\left(0.284 \mathrm{M}_{\mathrm{jup}}\right)$ also makes this hot \& inflated Saturn-mass planet an outstanding target for transmission spectroscopy. So far, $\mathrm{XO}-2 \mathrm{~b}$ is the only planet orbiting a metal-rich $\operatorname{star}([\mathrm{Fe} / \mathrm{H}] \sim 0.45$, Teske et al. (2015)) with the detection of both $\mathrm{Na}$ and $\mathrm{K}$ in its atmosphere (Sing et al. 2011; Pearson et al. 2019). Another hot-Saturn type planet WASP-49 b was also reported to have aerosol constraints (Cubillos et al. 2017) and neutral sodium at its high altitudes (Wyttenbach et al. 2017) of its extended atmosphere with cloud decks (Lendl et al. 2016). Only Mallonn et al. (2015) attempted at obtaining a transmission spectrum of HAT-P-19 with the OSIRIS spectrograph at the Gran Telescopio Canarias. They didn't find any trace of an additional absorption at any wavelength or any slope in their differential spectrophotometric search. With the same instrument, Sing et al. (2011) found a trace of potassium in the atmosphere of XO-2b. Nevertheless, HAT-P-19 b is still a promising target in terms of its potential of bearing heavy elements such as sodium and potassium, which may have escaped detection because of the pressure broadening in the planetary atmosphere (Mallonn et al. 2015). Whether such elements are found or not, this will provide another evidence for or against a suspected correlation between heavy element content in exoplanet atmospheres and planetary mass (Nikolov et al. 2018). These questions about the atmospheric content of this interesting planet, which is suggested as one of the prime targets to be observed with the James Webb Space Telescope (JWST) (Moliere et al. 2017), can be answered with the transmission spectroscopy observations from the space in the future. Therefore, determination of the planet and host star properties as a result of a detailed analysis of existing and new data with a holistic approach making use of recently developed analysis codes and global modelling techniques will help in characterization of its atmosphere too. Refining its ephemeris information is also crucial for planning future observations of the target with JWST and ground-based telescopes.

The system is also of particular interest due to the linear trend observed in its radial velocity residuals (Hartman et al. 2011), potentially indicating a gravitationally bound, yet unseen companion perturbing its orbit (Hartman et al. 2011; Seeliger et al. 2015) and/or causing the arrival times of the light from the system to the observer to change continuously (known as the Light Time Effect, LiTE). Nevertheless, studies of its transit timing variations (TTVs) (Seeliger et al. 2015; Maciejewski et al. 2018) have been inconclusive so far for the existence of such a potential third body. Only a few studies attempted to observe the target with high photometric precision to derive its parameters as well as its transit mid-times since its discovery back in 2011 (Hartman et al. 2011; Seeliger et al. 2015; Maciejewski et al. 2018). However, the system has been observed many times by amateur observers, as a result of which, at least a dozen moderate-quality light curves have been accumulated in the public archive of the Exoplanet Transit Database ${ }^{1}$, having the potential to be used in a TTV analysis. Transits of HAT-P-19 b have also been observed with TESS in the cycle-2 during sector 17.

We observed the target several times with the 1 meter Turkish telescope T100, located in the Bakırlitepe campus of the TÜBITAK National Observatory of Turkey (TUG) at an altitude of $2500 \mathrm{~m}$ above sea-level, near the south coast of the country; and achieved very high photometric precision thanks to the well-established telescope defocusing technique (Southworth et al. 2009; Baştürk et al. 2015). We made use of the same technique during two very precise transit observations of HAT-P-19 b with the Zeiss $1.23 \mathrm{~m}$ telescope in Calar Alto Observatory (CAHA), which is located

\footnotetext{
1 http://var2.astro.cz/ETD/
} 
in Spain at an altitude of $2170 \mathrm{~m}$. We analyzed the most precise transit light curves of our own as well as that from the literature (Hartman et al. 2011) acquired by ground-based facilities, the transit observations of TESS, and two occultation observations by Spitzer with the state-of-the-art second version of the EXOFAST software package (Eastman 2017, 2019), derived the global parameters of the system making use of the radial velocity observations by Hartman et al. (2011), and the atmospheric properties of the host star that we obtained from our own analysis of the Keck/HIRES archival spectra with primarily the iSpec software package (Blanco-Cuaresma et al. 2014; Blanco-Cuaresma 2019). We made use of the brightness of the target in different broadband filters and fit its spectral energy distribution based on its precise distance value thanks to Gaia mission to constrain the radius of its host star (Gaia Collaboration 2016, 2018). In addition, we collected all the light curves obtained so far by a number of observers around the world, all of which we corrected for the barycentre of our Solar System, measured mid-transit times in Dynamical Barycentric Julian Days (BJD-TDB), updated the ephemeris information for the system, established and analyzed the TTV diagram. We present the photometric and spectroscopic data that we used and the details of the data reduction procedure in Section 2. We provide the information on data analysis and present the parameters of the system as a result of the global modelling of the data, and the TTVs in Section 3. Finally we discuss the importance of our findings in the context of the hot-Saturn type planets, their formation, orbital evolution, and inflation of their atmospheres, as well as the potential reasons behind the observed TTVs in Section 4.

\section{OBSERVATIONS AND DATA REDUCTION}

\subsection{Photometric Data}

\subsubsection{TUG T100 Light Curves}

We observed eight transits of HAT-P-19 b between July 2014 and August 2019 with the $1 \mathrm{~m}$ Turkish Telescope TUG T100 and the high quality, cryo-cooler SI 1100 CCD with $4096 \times 4096$ pixels, which gives a field of view of $20^{\prime} \times 20^{\prime}$. All the observations were obtained in the Bessel- $R$ passband with 120 second integration time, which gave the best Signal-to-Noise Ratio (SNR) within an optimal number of observational points for each transit. We only changed the defocusing amount of the telescope (Southworth et al. 2009; Baştürk et al. 2015), hence the width of the Point Spread Function (PSF) from one night to another according to the sky quality.

We corrected our images with the AstroImageJ (hereafter AIJ) software package (Collins et al. 2017) for undesired instrumental effects by making use of the medians of 5-to-10 bias, dark, and twilight-sky flat images, shot during the same night as the observations. We made use of $2 \times 2$ pixel-binning to achieve a balance between the photometric precision and timing resolution, which decreases with the increase in the exposure times while the latter gets better since reading over a 4 times smaller number of pixels decreases the read-out time to 15 seconds within our setup. We converted all the observation timings to Dynamical Barycentric Julian Days (BJD-TDB) and recorded them in the head-

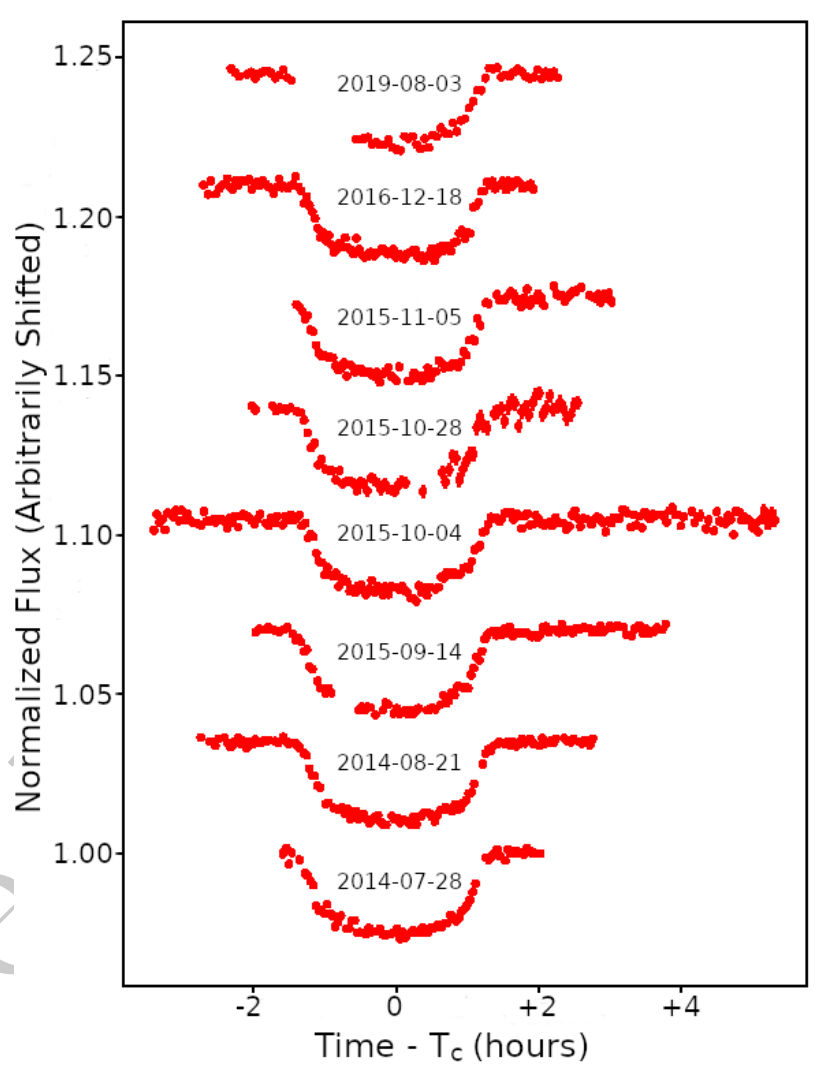

Figure 1. T100 light curves of HAT-P-19 b transits in Bessel- $R$ passband.

ers of the image files together with the calculated airmass values for those timings. AIJ makes it possible to perform ensemble aperture photometry (Honeycutt 1992) relative to a number of comparison stars. We used GSC 2283-1197, and 2MASS $00382684+3446556$ in the same field with HAT-P19, as our comparisons in the differential photometry, since their brightnesses are comparable and no photometric variations have been recorded so far in the timescales of our observations and sensitivity limits of our setup. Since our observations are defocused, we determined the centre of the apertures ourselves to avoid incorrect positions determined by the centroid method. We employed different aperture sizes for different nights with changing atmospheric seeing values. We then corrected for the airmass, and then normalized the relative fluxes determined by AIJ, by dividing them to the line fit to relative fluxes out of the transit profile. As a result, we obtained the normalized transit light curves for each of the nights of our observations with T100, which we present in the Fig. 1.

\subsubsection{CAHA Light Curves}

Light curves of two transits of the target were obtained using the $1.23 \mathrm{~m}$ telescope at the Calar Alto Observatory (CAHA), Spain, equipped with the DLR-MKIII CCD camera. This setup yielded a field of view of $21.5^{\prime} \times 21.5^{\prime}$ at a plate scale of $0.32^{\prime \prime}$ per pixel. We observed through Cousins $R$, as this filter gave the highest count rate for photons from the target star, and without binning or windowing. The telescope was defo- 
cussed in order to increase the photometric precision of the observations (Southworth et al. 2009) and using our standard approach for this and other telescopes (Mancini et al. 2014). Although we used autoguiding we found that the centroid of the target star drifted by roughly 10 pixels during the observations on both nights, but due to the high quality of the CCD and the use of telescope defocussing this had little impact on the results. We made use of $2 \times 2$ pixel-binning to achieve a balance between the photometric precision and timing resolution.

We reduced the data using the DEFOT code (Southworth et al. (2014) and references therein), which performs aperture photometry using the APER algorithm and allows for light curves to be obtained relative an optimally-weighted ensemble of comparison stars. We used three comparison stars for the night of 2014/09/02 and five for the night of 2014/09/06. The application of bias and flat-field calibrations had a negligible effect on the light curves except for a slight increase in noise, so we elected not to use these calibrations.

\subsubsection{TESS Light Curves}

We inferred the TESS light curve of HAT-P-19 b performing a maximum likelihood estimation of the flux of the target source as well as the fluxes of the neighbouring sources and the background emission. In order to extract the relative flux and uncertainty time-series, we performed point spread function (PSF) photometry on a $11 \times 11$ pixel region around HAT-P-19 in the full frame images of TESS. We used an empirical estimate of the Point Response Function $(\mathrm{PRF})^{2}$ of the TESS cameras that was measured while the instrument was operating in space. We obtained a PSF model as a function of sub-pixel shifts by regressing the super-sampled PRF to determine the coefficients of polynomial terms up to third order in sub-pixel shifts. For the background emission, we used a spatially flat template justified by the small region of interest.

We retrieved the source positions from TICv8 (Stassun et al. 2019) and corrected them for proper motion. We evaluated the model emission template of each point source by interpolating the PSF model at the respective subpixel shift. We assumed that the variance of a pixel is equal to the number of counts in the pixel and neglected interpixel covariances. Finally, we performed linear regression to determine the fluxes (i.e., template coefficients) of the target and neighbouring points sources and the background for all time bins.

We did not calculate the Photon Noise Rate (PNR), because the integration time for TESS ( 30 minutes) is comparable to the bin duration (30 ninutes) we made use of in the computation of the PNR, which we determined based on the ingress duration ( $\sim 22$ minutes). Although we attempted to calculate the $\beta$ factor, it was based only on 2 -point bins covering an hour of integration time giving values between 0.45-0.60 contradicting the level of red-noise in TESS data. Therefore we do not provide either of these noise statistics in Table 1. The red-noise component made it impossible for a single normalization level to be determined

2 https://archive.stsci.edu/tess/all_products.html for all TESS dataset. Hence, we cut the light curve into five segments, each of which has a transit profile at its centre, and we normalized these segments for the analysis to the out-of-transit flux level. Since the transit on 2019-10-18 had only a few points, we ignored it.

\subsubsection{Spitzer Light Curves}

We analyzed two occultation obseryations from the Spitzer space telescope in order to better constrain the orbit through $e \cos (\omega)$ parameter. Spitzer observations and light curve extraction were performed by Kammer et al. (2015) using the pixel-level decorrelation method described by Deming et al. (2015). Detailed information (bin size, aperture radius etc.) about the observations and photometry can be found in Table 2 of Kammer et al. (2015). We only analyzed two Spitzer occultation light curves at $3.6 \mu \mathrm{m}$ as presented by Kammer et al. (2015), who obtained two more occultation light curves at $4.5 \mu \mathrm{m}$ as well. However, they also constrained the prior on eclipse time based on the $3.6 \mu \mathrm{m}$ observations in their study, because the occultation depths at $4.5 \mu \mathrm{m}$ turned out to be significantly different than that observed at $3.6 \mu \mathrm{m}$. These light curves at $4.5 \mu \mathrm{m}$ have considerable scatter and the occultation is barely visible in only one of them. Therefore, we only used the two light curves at $3.6 \mu \mathrm{m}$ to constrain the $e \cos (\omega)$ parameter.

\subsection{Light Curve Selection for Global Modelling}

In order to select the best light curves in our sample for global analysis we made use of two well-defined metrics, Photometric Noise Rate (PNR) (Fulton 2011) which indicates the white noise and the $\beta$-factor which quantifies the red (or correlated) noise (Winn et al. 2008). PNR is defined by Fulton (2011) as the ratio of the standard deviation of the residuals to the median number exposures per minute including also the time spent for read-out for each observation. The $\beta$-factor, on the other hand, is the ratio of the average residuals in several bins to the standard deviation of the binned residuals. If only the white noise dominates the noise budget, then $\beta=1$. The ingress/egress timescale $(\tau)$ of HAT-P-19 b transits is $\sim 23$ minutes. Therefore, we grouped our light curve data points in variable sizes of bins from 13 to 33 minutes and took the median of $\beta$ factors of those 15 bins in total. Although the $\beta$ factors are comparable only within a group (Winn et al. 2008) of observation runs with the same telescope, two light curves we obtained with $1.23 \mathrm{~m}$ telescope of the Calar Alto Astronomical Observatory (CAHA) share similar characteristics with that performed with T100 in that they had been obtained in $R$ filters with similar transmission curves, exposing CCDs for long exposure times in the defocus mode. On the other hand, two light curves from the Fred Lawrence Whipple Observatory's (FLWO) $1.20 \mathrm{~m}$ telescope by Hartman et al. (2011) are in SDSS bands and we have also five TESS transit light curves of the target.

In all of our ground-based observations, the $\beta$ factor, which is a good indicator of the red noise, is between 0.45 and 1.83 , showing that white noise is the dominant noise source in all the observations. We had the largest values for this parameter on observations on 2015-10-04 and 28 with 
T100. The inferior quality of these light curves is also evident from other statistics, more strikingly from the PNR. Therefore, we did not select these light curves in the global modelling. We also had to eliminate the T100 observation on 2015-11-05, although the $\beta$ factor is only 0.85 , because the ingress is missing completely, which complicates the modelling as well as decreases the correlated noise artificially as pointed out by Winn et al. (2008). We also had to exclude the light curves acquired on 2016-12-18 transit due to the variations in atmospheric conditions just before the egress. Since these light curves have low-to-moderate quality, either the measured transit depths are significantly different from that observed in all other light curves, when the observing conditions were better or there is an insufficient number of data points at important orbital phases for modelling. Therefore, we decided not to include them in our analysis in obtaining the system parameters. However, it is still possible to determine the mid-transit times with good precision (on the order of a few seconds), hence we used this set of slightly inferior photometric quality observations in the analysis of transit timings. Timestamps in T100 FITS files are updated by the computer clock, which is synchronized with a GPS every few seconds. Therefore the errors in the measurements of mid-transit times are dominated by photometric precision rather than the timing.

We also computed the nightly average of the photometric measurement uncertainty $\left(\sigma_{\mathrm{ph}}\right)$ for each night and the root mean square errors of the linear fit to the out-of-transit segments used to detrend the light curves from changing airmass $\left(\sigma_{\mathrm{RMS}}\right)$. We observed that the transit depths are in one-standard-deviation for all the remaining light curves when these measurement errors are considered. As a result we decided to eliminate five T100 light curves and used the rest in the global modelling although one FLWO light curve (2009-12-01) has considerable scatter, therefore a low PNR. We provide a log of all 12 transit observations in total that we selected for global modelling in Table 1.

\subsection{Spectroscopic Data}

High resolution spectroscopic observations of HAT-P-19 were carried out with High Resolution Echélle Spectrometer (HIRES) attached on the $10 \mathrm{~m}$ Keck Telescope between October 2009 and March 2010 and the High-Dispersion Spectrograph (HDS) on the Subaru telescope on Mauna Kea by Hartman et al. (2011) to measure its radial velocity variations due to the transiting object, the planetary nature of which was revealed as a result. For our spectral analysis, we used the only available spectrum in the Keck/HIRES archive, that is free of the absorption lines of $\mathrm{I}_{2}$ gas due to the iodine cell used to increase radial velocity precision because HDS spectra from Subaru Telescope have narrow wavelength coverage and lower SNR. Average spectral resolution of the Keck/HIRES spectrum is $\mathrm{R} \sim 55000$, which is sufficient to determine the fundamental atmospheric properties of the host star. The spectrum covers a wide wavelength interval between 3360 - $8100 \AA$. An average SNR of $\sim 130$ was achieved in the 1390 second-exposure on 23 February 2010. This spectrum was also used by Hartman et al. (2011) for the same purpose. We also analyzed it to check and confirm the stellar parameters of the host-star and test their find- ings with different spectroscopic analysis tools, line lists, and stellar atmosphere models.

We corrected the spectrum for both the Doppler shift due to the orbital motion of the star about the common centre of mass with the planet HAT-P-19 b and the orbital motion of the Earth about the Sun. We also removed the cosmic rays, and cut the wavelength regions dominated by telluric lines and where the SNR is too low for a thorough analysis. We determined the continuum level from a synthetic spectrum that we used as a visual template for comparison because it is very challenging to determine it from the observational spectrum due to the numerous spectral lines which are blended in most cases. We then normalized the spectrum to this continuum level. We designed this synthetic spectrum in iSpec software package (Blanco-Cuaresma et al. 2014; Blanco-Cuaresma 2019) making use of the lines from Vienna Atomic Line Database (VALD, Piskunov et al. (1995); Kupka et al. (2000); Rybichikova et al. (2015)) scaled by the ATLAS9 model atmosphere (Castelli \& Kurucz 2004) and solar chemical abundances from Asplund et al. (2009), and the Synthe code (Kurucz 1993), which is embedded in the iSpec software package. The initial values of spectroscopic parameters were taken from Hartman et al. (2011). In the wavelength regions, where there are significant differences between the line strengths in the observed stellar spectrum and the synthetic spectrum, the extent of such a strong line was accounted for in the determination of the continuum. The continuum points were marked on the observed stellar spectrum and used as the nodes of cubic spline functions fit to them, and then the spectrum was normalized by dividing the entire spectrum with these spline functions.

\section{DATA ANALYSIS AND RESULTS}

\subsection{Spectroscopy}

For stars with effective temperatures lower than $5500 \mathrm{~K}$, techniques based on the measurements of the ratios of equivalent widths or depths of certain lines (mostly Fe-I and FeII) (Gray 1994), wings of the $\mathrm{H}_{\alpha}$ line (Fuhrmann 2004), and excitation/ionization balance (Santos et al. 2004) are not optimum if not totally inadequate in the determination of fundamental parameters due to the excessive line blending (Tsantaki et al. 2013). Synthetic spectrum fitting (Valenti \& Fischer 2005) is the preferred technique in this regime of stars; which has been our main method in deriving the fundamental parameters. We looked for the best fitting synthetic spectrum to our observational one with the least-squares minimization method by using the iSpec software package (Blanco-Cuaresma 2019). We tried to fit only the lines of Fe-I and Fe-II having 0.03 to 1.0 line depths, resulted in $\sim 1300$ lines which we further reduced to 626 in total (611 Fe-I and 15 Fe-II) after having eliminated lines with higher uncertainties than their equivalent widths (EW) and beyond the observed \pm 0.5 dex scatter in Fig. 2 about the mean iron abundance. iSpec also filters out the lines from its analysis, to which it fails to fit a Gaussian. Nevertheless, we still end up with somewhat blended lines that we ignored because it has been shown by Tsantaki et al. (2013) that fundamental parameters $\left(\mathrm{T}_{\text {eff }}, \log g,[\mathrm{Fe} / \mathrm{H}]\right)$ derived from an analysis based on only unblended lines to that involving the blended lines do not significantly differ if EWs 
Table 1. A log of photometric observations analyzed within this study. Nightly average of the photometric measurement uncertainties $\left(\sigma_{\mathrm{ph}}\right)$, RMS error of linear fits to out-of-transit levels $\left(\sigma_{\mathrm{RMS}}\right)$, Photon Noise Ratio (PNR), and the $\beta$-factors quantifying the white and red-noise, respectively are given in 4-7 columns.

\begin{tabular}{ccccccccc}
\hline $\begin{array}{c}\text { Obs. } \\
\text { Number }\end{array}$ & $\begin{array}{c}\text { Starting } \\
\text { Date }[\mathrm{UT}]\end{array}$ & Facility & $\begin{array}{c}\text { Exp.Time } \\
{[\mathrm{s}]}\end{array}$ & Filter & $\begin{array}{c}\sigma_{\mathrm{ph} .} \\
{[\mathrm{mmag}]}\end{array}$ & $\begin{array}{c}\sigma_{\mathrm{RMS}} \\
{[\mathrm{mmag}]}\end{array}$ & $\begin{array}{c}\text { PNR } \\
{[\mathrm{mmag}]}\end{array}$ & \begin{tabular}{c}
$\beta$ \\
\hline 1
\end{tabular} \\
\hline $2014-07-28$ & T100 & 120 & Bessel- $R$ & 1.03 & 1.27 & 0.83 & 0.89 \\
2 & $2014-08-21$ & T100 & 120 & Bessel- $R$ & 0.89 & 1.06 & 0.69 & 1.17 \\
3 & $2015-09-14$ & T100 & 120 & Bessel- $R$ & 1.10 & 1.27 & 0.83 & 1.11 \\
4 & $2014-09-02$ & CAHA & 80 & $R_{c}$ & 0.92 & 0.97 & 0.71 & 1.83 \\
5 & $2014-09-06$ & CAHA & 130 & $R_{c}$ & 0.72 & 0.85 & 0.53 & 1.73 \\
6 & $2009-10-30$ & FLWO & 150 & Sloan $i^{\prime}$ & 0.90 & 1.56 & 0.98 & 1.17 \\
7 & $2009-12-01$ & FLWO & 90 & Sloan $i^{\prime}$ & 1.13 & 3.01 & 2.46 & 1.59 \\
8 & $2019-10-10$ & TESS & 1800 & TESS & 0.42 & 1.38 & - & - \\
9 & $2019-10-14$ & TESS & 1800 & TESS & 0.42 & 1.28 & - \\
10 & $2019-10-22$ & TESS & 1800 & TESS & 0.42 & 1.21 & - & - \\
11 & $2019-10-26$ & TESS & 1800 & TESS & 0.42 & 1.11 & - & - \\
12 & $2019-10-30$ & TESS & 1800 & TESS & 0.41 & 0.92 & - & - \\
\hline
\end{tabular}

of iron lines are employed, especially for stars with effective temperatures lower than $5000 \mathrm{~K}$.

For an initial fit to the observed data, $\mathrm{T}_{\text {eff }}, \log g,[\mathrm{Fe} / \mathrm{H}]$, and line broadening (the sum of rotational and macroturbulent velocities) were adjusted. When a solution is converged, $\mathrm{T}_{\text {eff }},[\mathrm{Fe} / \mathrm{H}]$, and broadening amount (in velocity units) were fixed to the values found in the initial fit, while the surface gravity was adjusted to look for a convergence. Since the effect of $\log g$ on the spectra of cool dwarfs is marginal, it will be more adequate to determine its value by using the stellar density derived from the light curve analysis. In order to keep the surface gravity consistent with the mean density of the star, that we fixed to its value we derived from the light curve analysis for each output surface gravity value, we provided all the fundamental parameters to the equation given by Torres et al. (2010) as input to derive the stellar mass and radius. We compared the resultant stellar density to the empirical value that we get from the light curve analysis. When the difference between the two densities is minimized to $0.001 \mathrm{~g} \mathrm{~cm}^{-3}$, we accepted the current surface gravity as that of the host star and stopped the iteration, while other parameters were adjusted for a final convergence. The resultant atmospheric model gave the effective temperature, metallicity, and the broadenings (micro / macroturbulence, and the projected rotational velocity), which are summarized in the third column of Table 2 .

We measured equivalent widths of $611 \mathrm{Fe}-\mathrm{I}$ and $15 \mathrm{Fe}-$ II "clean lines", and determined the microturbulent velocity $\left(v_{\text {mic }}\right)$, for which the EWs become independent of the chemical abundances of the related species, and the scatter in the mean abundances for those is minimized. The value we find for this parameter in this manner is not too different for the microturbulent velocities assumed for HAT-P-19 $\left(v_{\text {mic }}=0.85 \mathrm{~km} / \mathrm{s}\right)$ in the literature based on the calibrations (Hartman et al. 2011; Brewer et al. 2016). However, we preferred to fix the microturbulent velocity to the value we have determined from the curve of growth $\left(v_{\text {mic }}=0.80\right.$ $\mathrm{km} / \mathrm{s}$ ) given in Fig. 2 .

We also attempted at deriving the effective temperature from the ionization balance between Fe-I and Fe-II. However, due to the low temperature of the target, and because there are not many lines of Fe-II in the spectrum with good SNR allowing precise measurements to be made, the effective temperature was underestimated by $\sim 200 \mathrm{~K}$. Hence,

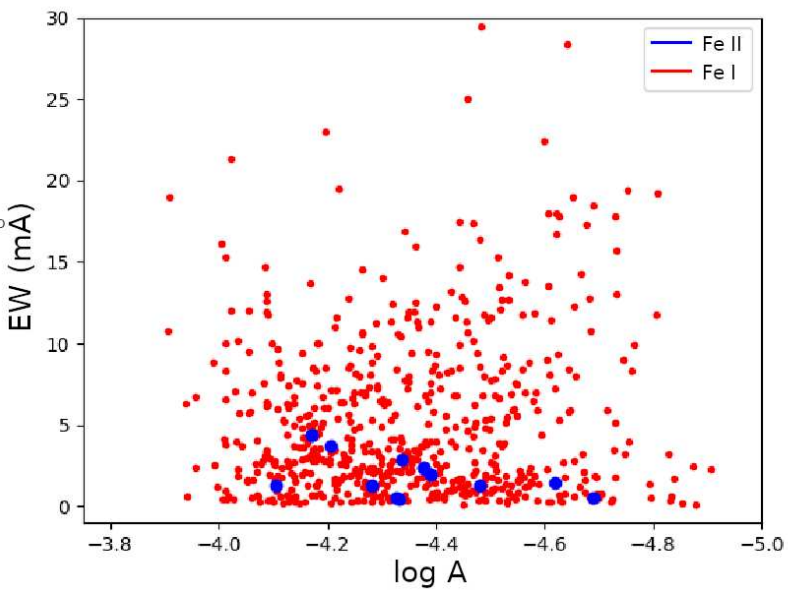

Figure 2. Equivalent widths of 611 Fe-I and 15 Fe-II lines with respect to their abundances for the microturbulent velocity $\left(\left(v_{m i c}=0.80 \mathrm{~km} / \mathrm{s}\right)\right.$ that minimizes the correlation between the two.

we rejected the analysis and decided to use the fundamental parameters from the synthetic spectrum fitting. This kind of analysis based on EW measurements gives reliable microturbulent velocities. That is why we used the $v_{\text {mic }}$ that we determined from this analysis but not the other parameters.

On the other hand, macroturbulent motions and stellar rotation similarly broaden the line profiles, shaping them to be Gaussians. Therefore, the broadening determined by the iSpec software package from the line profiles is the convolution of both broadening mechanisms. If one of these parameters (e.g. macroturbulence) is fixed to 0 , and the other is set free; then the latter (projected rotational velocity $(v$ sini) in our example) is responsible for the entire broadening. That is why we intended to use the typical macroturbulent velocity for our target. Since HAT-P-19 has an effective temperature that is very close to the borderline of the calibration given by Gilmore et al. (2012), we derived the macroturbulent velocity with both calibration equations, one of which is for stars above $T_{\text {eff }}=5000 \mathrm{~K}\left(v_{\operatorname{mac}}=3.35 \mathrm{~km} / \mathrm{s}\right)$ and the other below that limit $\left(v_{\text {mac }}=2.87 \mathrm{~km} / \mathrm{s}\right)$. We found an intermediate value, which is in agreement with the value we found from the weakest Fe-I lines that form deeper in 
Table 2. Atmospheric Parameters of HAT-P-19.

\begin{tabular}{lcccc}
\hline \hline Parameter & H11 & B16 & Initial Fit & Final Fit \\
\hline$T_{\text {eff }}[\mathrm{K}]$ & $4990 \pm 130$ & 4951 & $4988 \pm 54$ & $4991 \pm 50$ \\
$\log g[\mathrm{cgs}]$ & $4.54 \pm 0.05$ & 4.44 & $4.55 \pm 0.07$ & 4.53 \\
{$[\mathrm{Fe} / \mathrm{H}][\mathrm{dex}]$} & $0.23 \pm 0.08$ & 0.29 & $0.23 \pm 0.05$ & $0.24 \pm 0.05$ \\
$v \operatorname{sini}[\mathrm{km} / \mathrm{s}]$ & $0.7 \pm 0.5$ & 1.8 & $1.41 \pm 0.87$ & $0.88 \pm 1.09$ \\
$v_{\text {mac }}[\mathrm{km} / \mathrm{s}]$ & 2.81 & 1.8 & $2.82 \pm 0.59$ & 3.00 \\
$v_{\text {mic }}[\mathrm{km} / \mathrm{s}]$ & 0.85 & 0.85 & $0.79 \pm 0.15$ & 0.80 \\
\hline
\end{tabular}

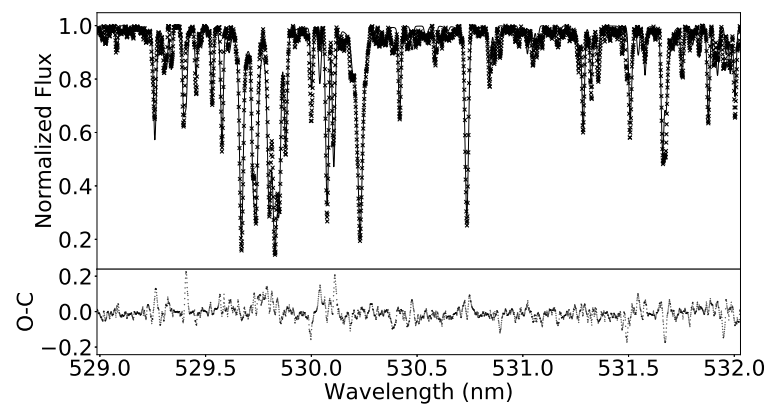

Figure 3. Keck/HIRES spectrum of HAT-P-19 (data points) and the best fit synthetic spectrum (continuous curve) with iSpec (5290 - $5320 \AA$ region).

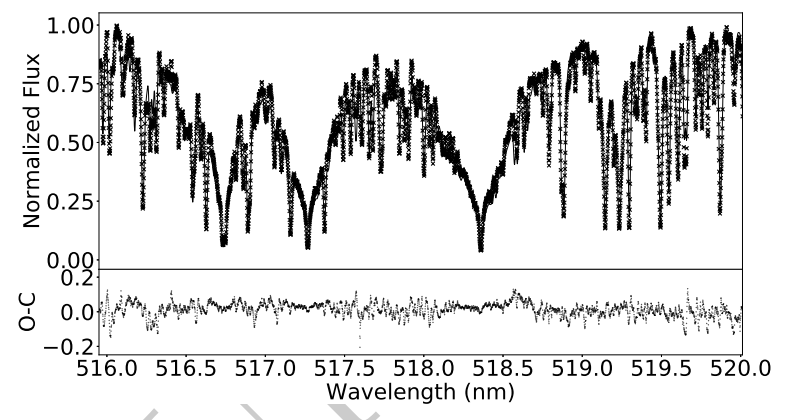

Figure 4. Keck/HIRES spectrum of HAT-P-19 (data points) and the best fit synthetic spectrum (continuous curve) with iSpec (5160 - $5200 \AA$ region).

the atmosphere $\left(v_{\operatorname{mac}}=3.00 \mathrm{~km} / \mathrm{s}\right)$, after having deconvolved the microturbulent velocity from the profiles. We then fixed the macroturbulent velocity to this value, and adjusted the projected rotational velocity and found it to be $v \sin i=0.88 \pm 1.09 \mathrm{~km} / \mathrm{s}$. The derived atmospheric parameters from our analysis of the Keck/HIRES spectra of HAT-P-19 are given in Table 2, in comparison with the results from previous works. Our results mostly agree with the previous findings (Hartman et al. 2011; Brewer et al. 2016) although different line lists, techniques, spectroscopic tools, solar abundances, and stellar atmosphere models have been employed. This agreement assured us about the reliability of host-star parameters for the determination of planetary parameters that depend on them. We provide the best synthetic spectrum for the observed in two different wavelength regions in Figs. 3 \& 4.

\subsection{Global Modelling}

We used the state-of-the-art, second version of EXOFAST (Eastman 2017, 2019) in order to obtain a global model of the light and radial velocity curves, stellar atmospheric parameters derived from our spectroscopic analysis, and the brightness of the host star in different passbands. We fitted our three best light curves from T100, two light curves from CAHA $1.23 \mathrm{~m}$ telescope, two light curves from FLWO $1.20 \mathrm{~m}$ observations (Hartman et al. 2011), and all five light curves from TESS that we selected due to their high quality, quantified by the PNR and the $\beta$ parameters as well as their completeness. We also employed two occultation observations of Spitzer made in $3.6 \mu \mathrm{m}$ to better constrain the $e \cos (\omega)$ parameter. We enforced Gaussian priors on the epoch $\left(R_{\mathrm{c}}=2456867.425742\right.$ in BJD-TDB) based on the mid-transit time of our first observation with T100, and orbital period $\left(\mathrm{P}_{\text {orb }}=4.0087826\right.$ days $)$ determined by Hartman et al. (2011). We used the stellar atmospheric parameters we determined as a result of our own analysis of one high resolution, high SNR Keck/HIRES spectrum, as $\mathrm{T}_{\mathrm{eff}}=4991 \pm 50 \mathrm{~K},[\mathrm{Fe} / \mathrm{H}]=0.24 \pm 0.05 \mathrm{dex}$, and $v \sin i=0.88 \pm 1.09 \mathrm{~km} / \mathrm{s}$ for the mean and standard deviation of Gaussian priors. We did not enforce a prior for the surface gravity since the light curve modelling of exoplanet transits provide better constraints for this parameter, which is not well constrained in spectroscopic analyses of cool star atmospheres. Limb darkening coefficients for the quadratic law were interpolated from the tables provided by Claret \& Bloemen (2011) during the light curve fitting. Cosine of the orbital inclination (cos i) was assigned to a uniform prior.

EXOFAST-v2 also models the Spectral Energy Distribution (SED) based on the passband brightnesses from broadband photometry and the stellar distance. We collected passband brightness values of HAT-P-19 from all-sky survey catalogues, which we list in Table 3 . We intended to use the Sloan ugriz magnitudes from the Sloan Digital Sky Survey (SDSS) catalogue. However, there are two different point sources listed in the catalogue for the coordinates of our target, while Gaia observations do not reveal a nearby companion to HAT-P-19. This might have been caused by two differing measurements at different times with SDSS most probably due to the saturation of the flux values in ADU units in one of the observations due to the brightness of the target. Therefore, we had to use $g^{\prime}, r^{\prime}, i^{\prime}$ magnitudes from APASS DR9 catalogue (Henden et al. 2016), while we made use of the $z P S$ from PAN-STARRS catalogue (Chambers et al. 2016) instead, because at least the upper limits for realistic errors have been listed in these catalogues for the passband brightnesses. Finally, we used $u^{\prime}$ magnitude from the original SDSS catalogue (Alam et al. 2015 ), since this value is the same for both point sources in the catalogue. We took the effective wavelengths of the filters used in the corresponding sky surveys from The Spanish Virtual Observatory (SVO) Filter Service (Rodrigo et al. 2012; Rodrigo \& Solano 2013), and provided them in Table 3.

Our analysis relies on the most precise distance of the object thanks to the exquisite parallax value provided by the Gaia mission (Gaia Collaboration 2016, 2018). We accounted for the systematic offset in Gaia parallaxes, noticed by Stassun \& Torres (2018), in deriving the distance of 
Table 3. Passband Brightnesses of HAT-P-19.

\begin{tabular}{|c|c|c|}
\hline Passband & $\overline{\lambda_{e f f}}$ & Magnitude \\
\hline \multicolumn{3}{|c|}{ SDSS (Alam et al. 2015) } \\
\hline SDSS $u^{\prime}$ & 3594.9 & $15.589 \pm 0.100$ \\
\hline \multicolumn{3}{|c|}{ APASS-DR9 (Henden et al. 2016) } \\
\hline Johnson B & 4378.1 & $14.834 \pm 0.051$ \\
\hline Johnson V & 5466.1 & $12.853 \pm 0.050$ \\
\hline SDSS g' & 4640.4 & $13.381 \pm 0.176$ \\
\hline SDSS r' & 6122.3 & $12.500 \pm 0.043$ \\
\hline SDSS i' & 7439.5 & $12.275 \pm 0.174$ \\
\hline \multicolumn{3}{|c|}{ Pan-STARRS (Chambers et al. 2016) } \\
\hline zPS & 8657.8 & $12.18 \pm 0.05$ \\
\hline \multicolumn{3}{|c|}{ 2MASS (Cutri et al. 2003) } \\
\hline$J_{2 M A S S}$ & 12350.0 & $11.095 \pm 0.020$ \\
\hline$H_{2 M A S S}$ & 16620.0 & $10.644 \pm 0.022$ \\
\hline$K_{2 M A S S}$ & 21590.0 & $10.546 \pm 0.019$ \\
\hline \multicolumn{3}{|c|}{ All WISE (Cutri et al. 2014) } \\
\hline WISE1 & 33526.0 & $10.495 \pm 0.022$ \\
\hline WISE2 & 46028.0 & $10.557 \pm 0.020$ \\
\hline WISE3 & 115608.0 & $10.561 \pm 0.091$ \\
\hline \multicolumn{3}{|c|}{ NOMAD (Zacharias et al. 2005) } \\
\hline Johnson R & 6695.6 & $11.99 \pm 0.1$ \\
\hline
\end{tabular}

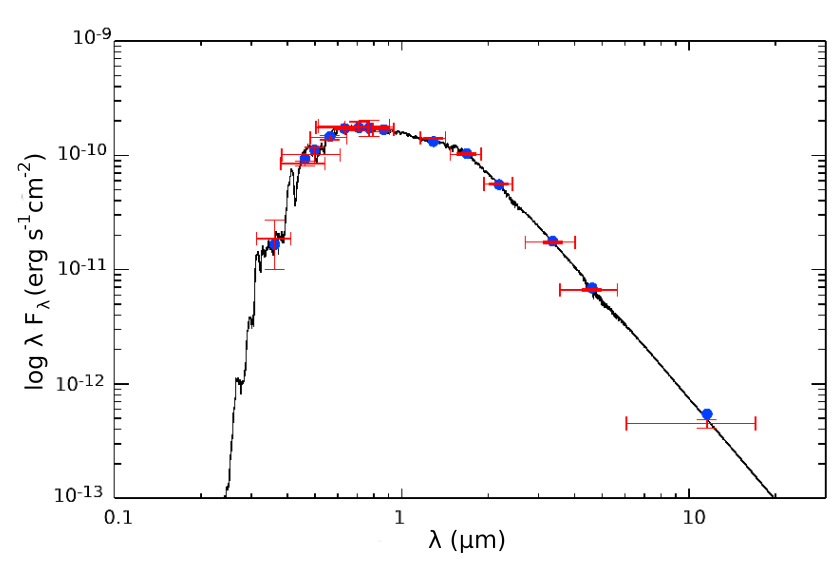

Figure 5. The passband brightnesses are represented with red data points, the error bars of which in wavelength show the widths of the corresponding filters while those in flux denote the measurement uncertainties. The black continuous curve is the model SED, while blue circles are broadband averages on the model.

HAT-P-19 and added 0.082 miliarcseconds to the Gaia parallax value, and 0.033 to its uncertainty; which translates into a $-3.332 \mathrm{pc}$ difference in the distance of the object and $+1.271 \mathrm{pc}$ in its uncertainty, and had $d=199.921 \pm 2.738$ pc as a result. EXOFAST constrains the V-band extinction $\left(A_{V}\right)$ based on its SED model (Fig. 5 for HAT-P-19), which is a measure of the star's bolometric flux and the ratio of the stellar radius to its distance. Having a precise and independent measurement of the distance, therefore, makes the constraint on the stellar radius very strong through luminosity relation. During the global modelling, the extinction coefficient $\left(A_{V}\right)$ was adjusted but limited by the maximum extinction value to that given by the maps from Schlegel et al. (1998).

EXOFAST-v2 can fit a MESA isochrone (MIST) (Paxton et al. 2011, 2013, 2015) and find the position of the host star on the stellar evolutionary tracks based on

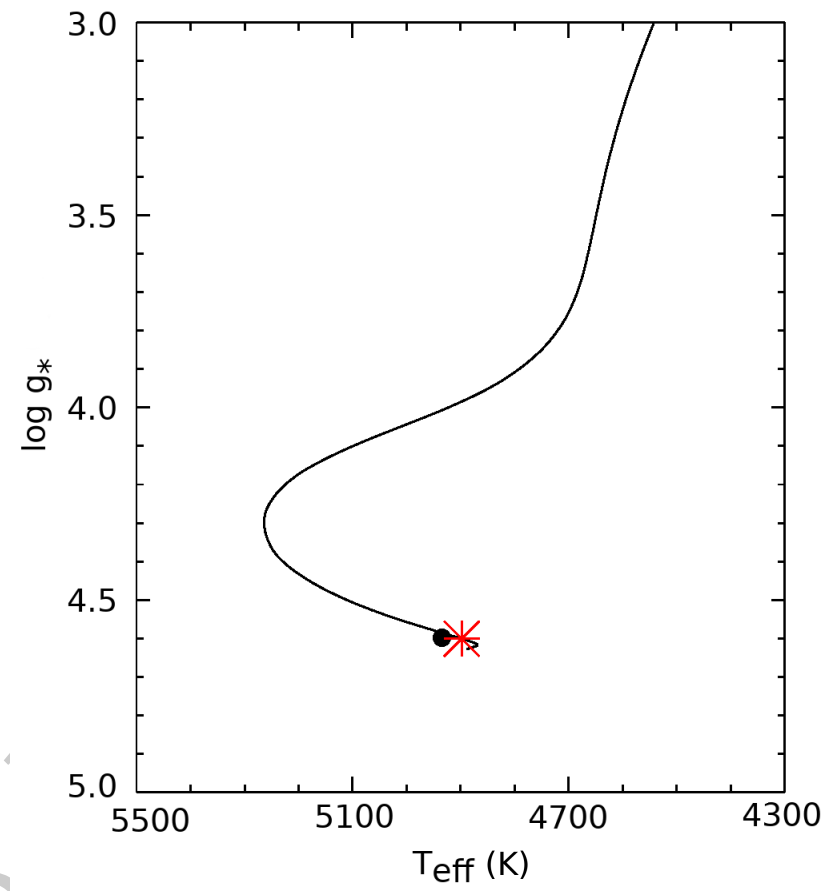

Figure 6. The best fitting MIST track, interpolated at the model values for $M_{*},[\mathrm{Fe} / \mathrm{H}]_{0}$ and $E E P$, is given by the black continuous curve. Red asterisk symbol indicates the position along the best fitting MIST track for HAT-P-19, while the black circle is at the model value for $T_{\text {eff }}$ and $\log g_{*}$.

a trilinear interpolation in Equivalent Evolutionary Phase parameter (EEP), which quantifies the phase at which the host star in its evolutionary history (Eastman 2019), initial stellar mass $\left(M_{*}\right)$ and initial metallicity $\left([\mathrm{Fe} / \mathrm{H}]_{0}\right)$ parameters, which are the mass and surface metallicity value at the zero-age main-sequence $(\mathrm{EEP}=202)$. From the corresponding MESA stellar track, the code derives the effective temperature $\left(\mathrm{T}_{\text {eff }}\right)$, stellar radius $\left(R_{*}\right)$, and surface metallicity $([\mathrm{Fe} / \mathrm{H}])$; and compares them to the model values at the current MCMC step. Since we have a well-constrained $\mathrm{T}_{\text {eff }}$ $[\mathrm{Fe} / \mathrm{H}]$ pair from spectroscopy, and $R_{*}$ from SED fitting, the MCMC algorithm constrains the position of the host star on $\mathrm{T}_{\text {eff }}-\log g$ plane by changing $M_{*}$, and $[\mathrm{Fe} / \mathrm{H}]_{0}$ to match the values of these parameters by keeping $M_{*}$ consistent with that found from the stellar density $\left(\rho_{*}\right)$. The best-fitting MIST evolutionary track for HAT-P-19 from our analysis is given in Fig. 6 with the black continuous curve.

Then the posterior distributions of the global model parameters were calculated by employing likelihood functions utilizing the goodness of fit estimators $\left(\chi^{2}\right)$ from a run of 200 chains of 50000 iterations. When the variance between the iterations became smaller than the variation inherent to the parameter value, the iterations were aborted by the program, making use of the Gelman-Rubin statistics for the purpose. We provide the median values of all parameters, distributions of which were determined from the global modelling of three T100, two CAHA, two FLWO (Hartman et al. 2011), and five TESS light curves, radial velocity values derived from Keck/HIRES and Subaru / HDS observations by Hartman et al. (2011), available passband brightnesses of the target from broadband photometry, Gaia distance 


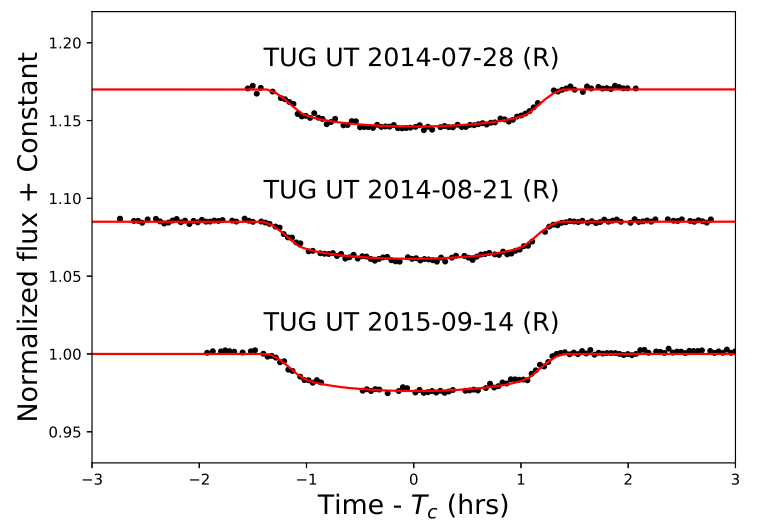

Figure 7. Three selected individual T100 transit observations (black data points) and the EXOFAST-v2 models (red continuous line) to each of these light curves based on the parameters in Table 3.2 .

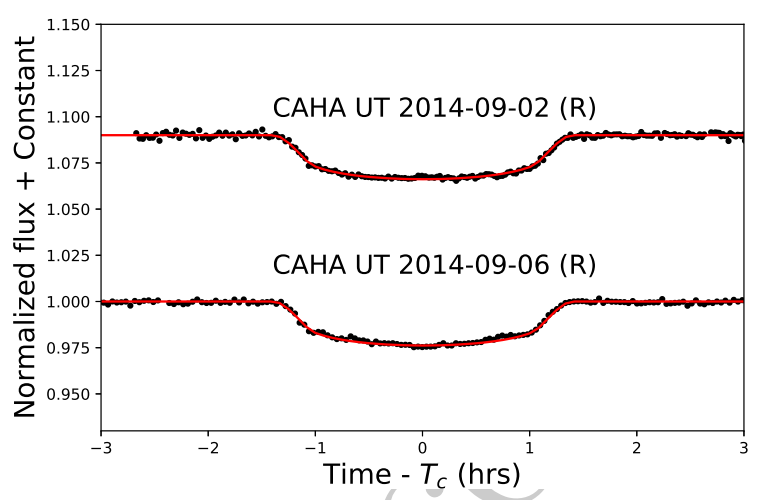

Figure 8. The same as Fig. 7 but for the two CAHA $1.23 \mathrm{~m}$ light curves.

corrected for the systematic shift, and stellar atmospheric parameters determined from our own spectroscopic analysis in Table 3.2. All the light curves used in the global modelling are given separately in Figs.7, 8, 9, 10, and 11.

EXOFAST-v2 models the radial velocity together with the light curves, and then determines the orbital parameters from a global fit. We took the radial velocity values and their errors from Hartman et al. (2011) as they are given, and fitted a velocity offset for each of the HIRES and HDS datasets within every MCMC chain. We also allowed a linear drift in the systemic velocity of HAT-P-19 and fitted a Keplerian model. The linear trend in the radial velocities before phasing the observations and the model is clear and obvious also in our global model in Fig. 12. We also present the radial velocity data points and the Keplerian model phased with the periastron passage, mid-transit time and the orbital period so that the minimum of the radial velocities is at 0.5 orbital phase in Fig. 13. A possible explanation of the linear increase in the radial velocities of HAT-P-19 could be an unseen third body gravitationally bound to the system, which would lead to TTVs, that we discuss in the next section.

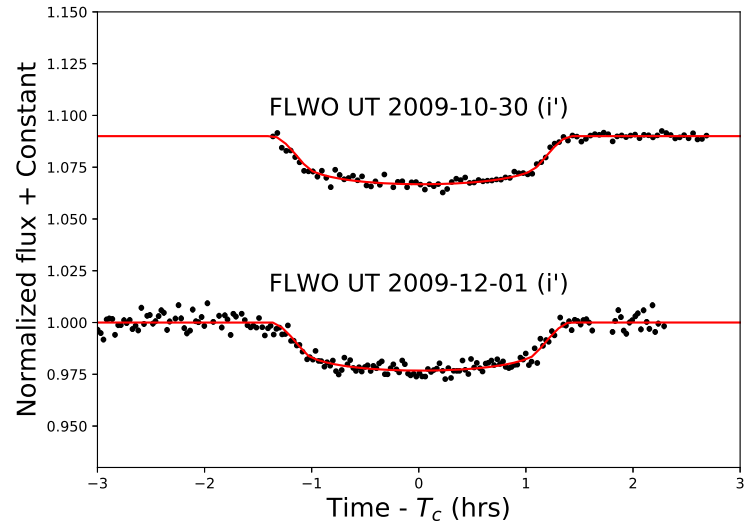

Figure 9. The same as Fig. 7 but for the two FLWO 1.23 light curves obtained by Hartman et al. (2011).

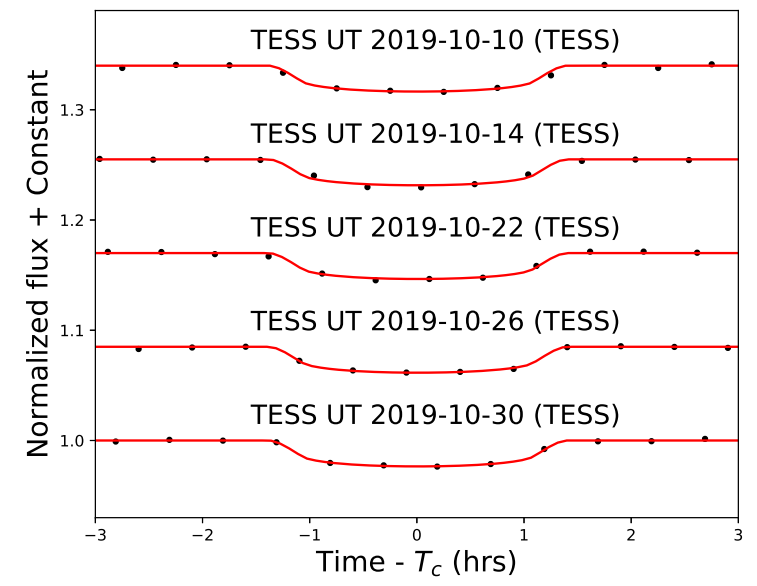

Figure 10. The same as Fig. 7 but for the five TESS light curves.

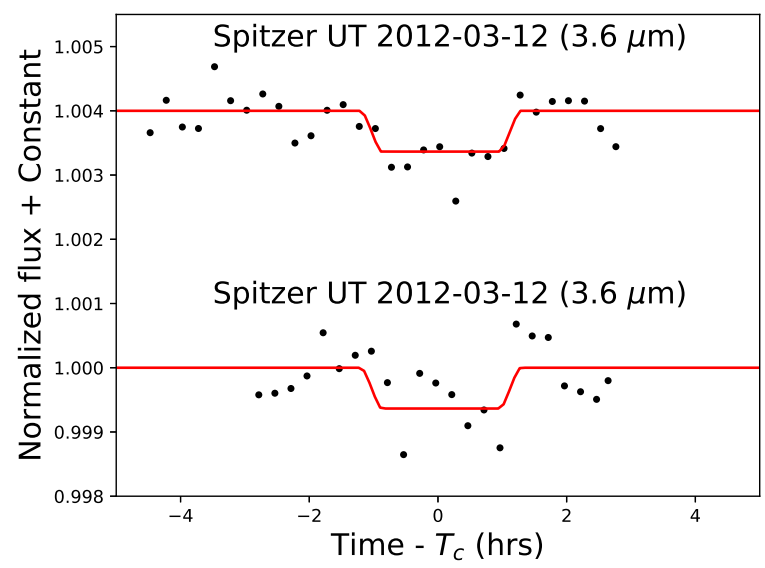

Figure 11. The same as Fig. 7 but for the Spitzer occultation observations in $3.6 \mu \mathrm{m}$. 
Table 4. Median values and 1 standard deviations for the parameters of the star HAT-P-19, and its exoplanet HAT-P-19 b.

\begin{tabular}{|c|c|c|}
\hline Symbol & Parameter (Unit) & Value \\
\hline \multicolumn{3}{|c|}{ Stellar Parameters: } \\
\hline$M_{*}$ & Mass $\left(M_{\odot}\right)$ & $0.845_{-0.035}^{+0.037}$ \\
\hline$R_{*}$ & Radius $\left(R_{\odot}\right)$ & $0.792_{-0.025}^{+0.021}$ \\
\hline$L_{*}$ & Luminosity $\left(L_{\odot}\right)$ & $0.344_{-0.025}^{+0.025}$ \\
\hline$\rho_{*}$ & Density (cgs) & $2.39_{-0.16}^{+0.25}$ \\
\hline $\log g$ & Surface gravity (cgs) & $4.567_{-0.022}^{+0.030}$ \\
\hline$T_{\text {eff }}$ & Effective Temperature (K) & $4969_{-48}^{+47}$ \\
\hline$[\mathrm{Fe} / \mathrm{H}]$ & Metallicity (dex) & $0.243 \pm 0.049$ \\
\hline$[\mathrm{Fe} / \mathrm{H}]_{0}$ & Initial Metallicity & $0.221_{-0.054}^{+0.055}$ \\
\hline Age & Age (Gyr) & $5.1_{-3.5}^{+4.8}$ \\
\hline$A_{V}$ & V-band extinction (mag) & $0.170_{-0.066}^{+0.054}$ \\
\hline$d$ & Distance (pc) & $199.921 \pm 2.738$ \\
\hline \multicolumn{3}{|c|}{ Planetary Parameters: } \\
\hline$P$ & Period (days) & $4.00878330 \pm 0.00000033$ \\
\hline$R_{\mathrm{p}}$ & Radius $\left(R_{\mathrm{J}}\right)$ & $1.064_{-0.034}^{+0.031}$ \\
\hline$M_{\mathrm{p}}$ & $\operatorname{Mass}\left(M_{\mathrm{J}}\right)$ & $0.284_{-0.017}^{+0.017}$ \\
\hline$T_{C}$ & Time of conjunction (BJD - TDB) & $2455135.63130_{0.00016}^{0.00017}$ \\
\hline$T_{0}$ & Optimal conjunction Time (BJD - & $2456827.337856 \pm 0.0 .000085$ \\
\hline$a$ & Semi-major axis (au) & $0.04699_{-0.00065}^{+0.00067}$ \\
\hline$i$ & Inclination (Degrees) & $89.11_{-0.29}^{+0.42}$ \\
\hline$e$ & Eccentricity & $0.024_{-0.022}^{+0.040}$ \\
\hline$\omega_{*}$ & Argument of Periastron (Degrees) & $-88.7_{-23.0}^{+6.9}$ \\
\hline$e \cos \omega_{*}$ & & $0.0007_{-0.0016}^{+0.0017}$ \\
\hline$T_{e q}$ & Equilibrium temperature $(\mathrm{K})$ & $986_{-18}^{+16}$ \\
\hline$K$ & RV semi-amplitude $(\mathrm{m} / \mathrm{s})$ & $40.7_{-2.1}^{+2.1}$ \\
\hline$\rho_{\mathrm{p}}$ & Density (cgs) & $0.293_{-0.026}^{+0.030}$ \\
\hline $\log _{\mathrm{p}}$ & Surface gravity & $2.795 \pm 0.032$ \\
\hline$\Theta$ & Safronov Number & $0.0295 \pm 0.0017$ \\
\hline$\langle F\rangle$ & Incident Flux $\left(10^{9} \mathrm{erg} \mathrm{s}^{-1} \mathrm{~cm}^{-2}\right)$ & $0.215_{-0.015}^{+0.014}$ \\
\hline \multicolumn{3}{|c|}{ Transit Parameters: } \\
\hline$b$ & Transit impact parameter & $0.204_{-0.097}^{+0.069}$ \\
\hline & Transit depth (fraction) & $0.01908_{-0.00021}^{+0.00025}$ \\
\hline$\delta_{S, 3.6 \mu}$ & Blackbody occultation depth at $3.6 \mu \mathrm{m}$ (ppm) & $401_{-27}^{+25}$ \\
\hline$a / R_{*}$ & Semi-major axis in stellar radii & $12.66_{-0.20}^{+0.43}$ \\
\hline$\tau$ & Ingress/egress transit duration (days) & $0.01462_{-0.00052}^{+0.00060}$ \\
\hline$T_{14}$ & Total transit duration (days) & $0.11578_{-0.00050}^{+0.00057}$ \\
\hline$\tau_{S}$ & Ingress/egress occultation duration (days) & $0.01389_{-0.00094}^{+0.00070}$ \\
\hline$T_{S, 14}$ & Total occultation duration (days) & $0.1107_{-0.0079}^{+0.0050}$ \\
\hline$T_{S}$ & Time of occultation (BJD - TDB) & $2455137.6375_{-0.0041}^{+0.0043}$ \\
\hline \multicolumn{3}{|c|}{ Wavelength Parameters: } \\
\hline$u_{1, R}$ & linear limb-darkening coefficient in $\mathrm{R}$ & $0.550 \pm 0.017$ \\
\hline$u_{2, R}$ & quadratic limb-darkening coefficient in $\mathrm{R}$ & $0.163 \pm 0.022$ \\
\hline$u_{1, i}$ & linear limb-darkening coefficient in i & $0.083 \pm 0.035$ \\
\hline$u_{2, i}$ & quadratic limb-darkening coefficient in i & $0.196 \pm 0.035$ \\
\hline$u_{1, T E S S}$ & linear limb-darkening coefficient in TESS Band & $0.447 \pm 0.027$ \\
\hline$u_{2, T E S S}$ & quadratic limb-darkening coefficient in TESS Band & $0.182 \pm 0.033$ \\
\hline
\end{tabular}




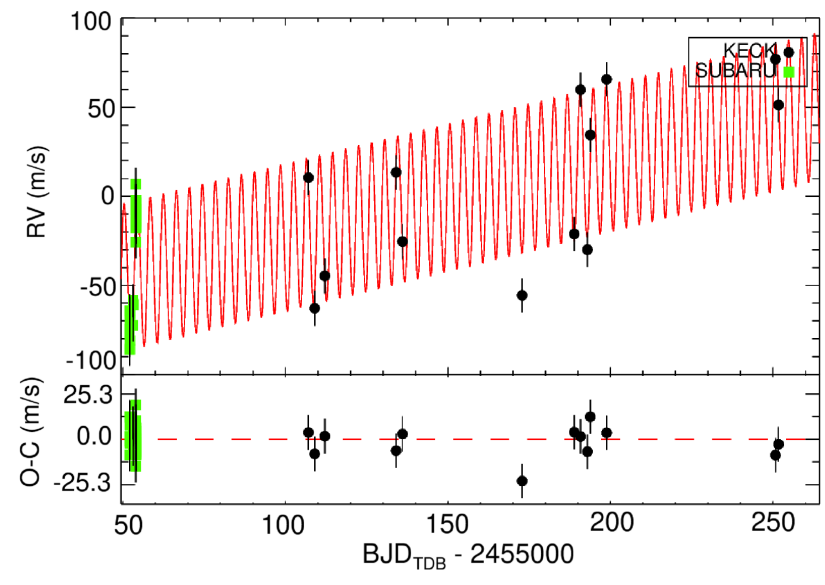

Figure 12. Radial velocity observations from Keck (black data points), and Subaru (green data points) as given by Hartman et al. (2011). The red curve represents our Keplerian best-fitting model.

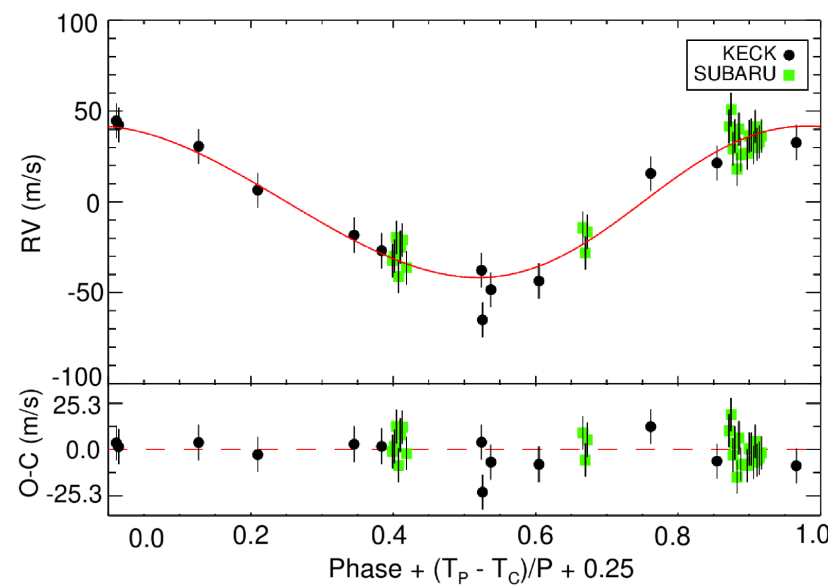

Figure 13. The same as Fig. 12 but with respect to the orbital phase and the RV drift is removed.

\subsection{Transit Timing Variations}

We collected light curve data for HAT-P-19 b transits from the literature and the Exoplanet Transit Database (ETD, http://var2.astro.cz/ETD/), where observations of amateur astronomers are also published. We contacted all the observers with quality transit light curves (indicated with a data quality index 2 out of 5 or better (1) as given in ETD). Based on these communications, we only included light curves from observers, who made use of either a GPS device or a timing server to coordinate their timings throughout their observations, and who provided the time reference for their observations (geocentric JD-UTC, HJD, BJD-UTC, etc.). In the absence of such information, we asked for the raw data from the observer, reduced them ourselves, computed and compared the light curves and the mid-transit times that we obtained with that given in the ETD by the observer. We converted the timings of all observations from the timing reference frames in which they were recorded to Dynamic Barycentric Julian days (BJD-TDB) by using our own scripts that we developed based on the relevant modules and functions of the astropy package (Astropy Collaboration

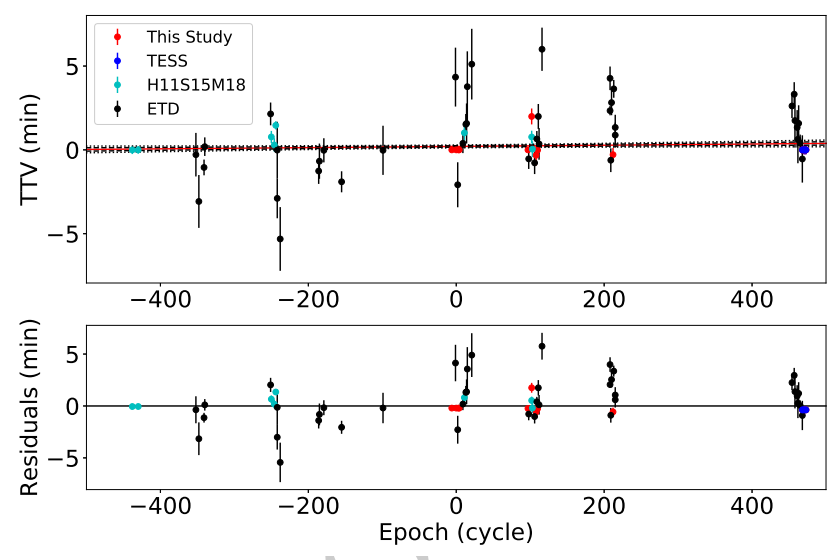

Figure 14. Difference between the observed and calculated mid-transit times with respect to that measured from our own observation with T100 (2014-08-21, EME: 2456891.47839(9)) and the orbital period from our global modelling $\left(P_{\text {orb }}=\right.$ 4.00878330(33) days), our linear fit (red continuous) and its 1 (dashed) and 2 (dotted) standard deviations, based on ETD (black), T100 \& CAHA (red), TESS (blue), and other literature data (cyan) from H11:Hartman et al. (2011), S15: Seeliger et al. (2015), M18:Maciejewski et al. (2018).

2013 , 2018). As a result, we had a homogenous set of midtransit times covering almost 10 years (889 orbital periods) since the discovery of the exoplanet HAT-P-19 b.

We then measured the mid-transit times of all the light curves that we collected (summarized in Table 5) from the literature and ETD, as well as all the light curves we used for modelling, and five additional T100 light curves that we did not use to determine the parameters due to relatively larger photometric errors and interruptions during the observations. We made use of the first EXOFAST version (Eastman 2017) to fit all these light curves separately to measure the mid-transit times in BJD-TDB, forming a homogeneous data-set from a heterogeneous set of observations. Then we computed the differences between observed and expected times of mid-transits based on the mid-transit time we measured from our best light curve with the minimum scatter (light curve recorded on 2014-08-21 (number 2), see Fig. 1 and Table 1) and the orbital period from our global modelling. We then plotted these differences from 65 light curves in total with respect to cycle number (epoch) of each observed transit before and after the reference midtime, and had the so-called TTV diagram that we provide in Fig. 14.

We took random, equally probable samples from a parameter space of linear coefficients (slope and y-intercept) and computed the likelihood of a linear fit with these coefficients to the TTV diagram. As a result we obtained the posterior probabilities for the parameters, corresponding to the change in reference mid-transit time $(\Delta T)$ and the orbital period $(\Delta P)$ within an MCMC run, involving 5000 number of iterations and 500 walkers. We discarded the first 500 steps (the so-called burn-in period) in each of the random walkers until an equilibrium is settled in the search. We refined the ephemeris information with the median values from our MCMC analysis based on all accepted data 
Table 5. Mid-transit times measured by us from our light curves, that from the literature and the Exoplanet Transit Database (ETD).

\begin{tabular}{|c|c|c|c|c|}
\hline $\begin{array}{l}\text { Mid-Transit Time } \\
\text { (BJD-TDB) }\end{array}$ & $\begin{array}{l}\text { Error } \\
\text { (days) }\end{array}$ & Filter & $\begin{array}{l}\text { Observer / } \\
\text { Reference }\end{array}$ & $\begin{array}{l}\text { RMS of Transit Fit } \\
\text { (Norm. Flux Units) }\end{array}$ \\
\hline 2455135.63130 & 0.00017 & Sloan $i$ & Hartman et al. (2011) & 0.0016 \\
\hline 2455167.70157 & 0.00016 & Sloan $i$ & Hartman et al. (2011) & 0.0030 \\
\hline 2455480.38647 & 0.00090 & Clear & Lomoz F. & 0.0053 \\
\hline 2455496.41966 & 0.00110 & $R_{c}$ & Naves R. & 0.0035 \\
\hline 2455524.48256 & 0.00033 & Clear & Muler G. & 0.0020 \\
\hline 2455528.49220 & 0.00039 & Clear & Ruiz J. & 0.0027 \\
\hline 2455885.27527 & 0.00047 & Clear & Ayiomamitis A. & 0.0034 \\
\hline 2455889.27840 & 0.00025 & Bessel R & Seeliger et al. (2015) & 0.0019 \\
\hline 2455905.31418 & 0.00028 & Bessel R & Seeliger et al. (2015) & 0.0021 \\
\hline 2455913.33317 & 0.00017 & $R_{c}$ & Seeliger et al. (2015) & 0.0012 \\
\hline 2455921.35082 & 0.00083 & $R_{c}$ & Naves R. & 0.0027 \\
\hline 2455921.35282 & 0.00118 & Clear & Corfini G. & 0.0031 \\
\hline 2455937.38427 & 0.00132 & $R_{c}$ & Naves R. & 0.0047 \\
\hline 2456145.84382 & 0.00053 & Clear & Shadic S. & 0.0037 \\
\hline 2456149.85301 & 0.00073 & Clear & Shadic S. & 0.0047 \\
\hline 2456173.90616 & 0.00050 & Clear & Garlitz J. & 0.0040 \\
\hline 2456270.11566 & 0.00044 & $R_{c}$ & Liyun Z. & 0.0028 \\
\hline 2456494.60882 & 0.00102 & Clear & Gonzalez J. & 0.0049 \\
\hline 2456867.42569 & 0.00009 & Bessel R & 100 (this study) & 0.0013 \\
\hline 2456887.47262 & 0.00122 & $\mathrm{~V}$ & Horta F. G. & 0.0046 \\
\hline 2456891.47839 & 0.00009 & Bessel R & T100 (this study) & 0.0011 \\
\hline 2456899.49451 & 0.00093 & $R_{c}$ & Barbieri L. & 0.0069 \\
\hline 2456903.50474 & 0.00011 & $R_{c}$ & CAHA (this study) & 0.0010 \\
\hline 2456907.51352 & 0.00011 & $R_{c}$ & CAHA (this study) & 0.0008 \\
\hline 2456927.55771 & 0.00041 & $R_{c}$ & Gillier C. & 0.0018 \\
\hline 2456943.59363 & 0.00043 & Clear & Benni P. & 0.0043 \\
\hline 2456935.56993 & 0.00033 & Bessel R & Seeliger et al. (2015) & 0.0025 \\
\hline 2456947.60245 & 0.00083 & $D$ & Horta F. G. & 0.0025 \\
\hline 2456951.61276 & 0.00146 & $I_{c}$ & Shadic S. & 0.0099 \\
\hline 2456975.66639 & 0.00146 & $I_{c}$ & Shadic S. & 0.0099 \\
\hline 2457280.33037 & 0.00009 & Bessel R & T100 (this study) & 0.0013 \\
\hline 2457284.33878 & 0.00042 & Clear & Ogmen Y. & 0.0030 \\
\hline 2457300.37482 & 0.00026 & Clear & Maciejewski et al. (2018) & 0.0020 \\
\hline 2457300.37567 & 0.00033 & Bessel R & T100 (this study) & 0.0016 \\
\hline 2457304.38310 & 0.00033 & Clear & Maciejewski et al. (2018) & 0.0019 \\
\hline 2457316.40888 & 0.00046 & $R_{c}$ & Salisbury M. & 0.0022 \\
\hline 2457324.42676 & 0.00042 & Bessel R & T100 (this study) & 0.0021 \\
\hline 2457328.43622 & 0.00033 & Clear & Bretton M. & 0.0016 \\
\hline 2457332.44454 & 0.00029 & Bessel R & T100 (this study) & 0.0014 \\
\hline 2457336.45472 & 0.00052 & Clear & Bretton M. & 0.0025 \\
\hline 2457340.46237 & 0.00066 & Clear & Molina D. & 0.0027 \\
\hline 2457356.50142 & 0.00090 & Clear & Bretton M. & 0.0031 \\
\hline 2457725.30694 & 0.00019 & Clear & Bretton M. & 0.0013 \\
\hline 2457725.30828 & 0.00049 & Clear & Bretton M. & 0.0020 \\
\hline 2457729.31367 & 0.00049 & Clear & Bretton M. & 0.0022 \\
\hline 2457729.31512 & 0.00068 & Clear & Bretton M. & 0.0022 \\
\hline 2457733.32484 & 0.00052 & Clear & Bretton M. & 0.0023 \\
\hline 2457745.35176 & 0.00037 & Clear & Girardin E. & 0.0061 \\
\hline 2457753.36741 & 0.00052 & Clear & Bretton M. & 0.0032 \\
\hline 2457753.36773 & 0.00052 & Clear & Bachschmidt, M. & 0.0032 \\
\hline 2458699.43930 & 0.00270 & Bessel R & T100 (this study) & 0.0006 \\
\hline 2458711.46783 & 0.00050 & Clear & Jongen, Y. & 0.0024 \\
\hline 2458723.49466 & 0.00050 & Clear & Raetz, M. & 0.0024 \\
\hline 2458727.50236 & 0.00112 & Clear & Pintr, P. & 0.0227 \\
\hline 2458739.52842 & 0.00072 & Clear & Deldem, M. & 0.0056 \\
\hline 2458743.53671 & 0.00099 & Clear & Jongen, Y. & 0.0959 \\
\hline 2458747.54616 & 0.00075 & Clear & Grunge, $\mathrm{T}$. & 0.0058 \\
\hline 2458755.56291 & 0.00035 & Clear & Jongen, Y. & 0.0019 \\
\hline 2458767.58860 & 0.00098 & Clear & Jongen, Y. & 0.0326 \\
\hline 2457741.34025 & 0.00028 & Bessel R & T100 (this study) & 0.0014 \\
\hline 2458767.58897 & 0.00018 & TESS Band & TESS (this study) & 0.0014 \\
\hline 2458771.59776 & 0.00018 & TESS Band & TESS (this study) & 0.0013 \\
\hline 2458779.61532 & 0.00018 & TESS Band & TESS (this study) & 0.0013 \\
\hline 2458783.62411 & 0.00018 & TESS Band & TESS (this study) & 0.0011 \\
\hline 2458787.63289 & 0.00018 & TESS Band & TESS (this study) & 0.0009 \\
\hline
\end{tabular}




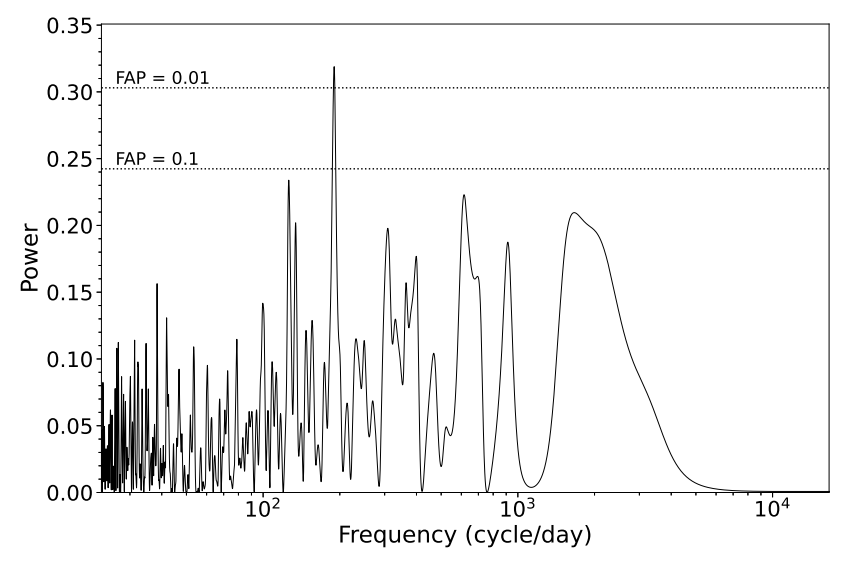

Figure 15. Lomb-Scargle Periodogram of TTV residuals from the linear fit in Fig-14. False Alarm Probabilities (FAP) of 0.1 and 0.01 are indicated as dotted horizontal lines.

satisfying the PNR and $\beta$ thresholds from the ETD, literature, and our light curves (Eq.1).

\section{$T_{c}(\mathrm{BJD}-\mathrm{TDB})=2456891.47853(3)+4^{d} .0087835(13) \times E$}

We also searched for a periodicity in the frequency space (Fig. 15) and found one frequency with significantly low False Alarm Probability (FAP 0.005) corresponding to a period of 190 days. This frequency arises from seasonal sampling frequency of the observations especially due to the data gaps in data with relatively smaller observational errors, which can be noticed when the data is phased with respect to this frequency. When we remove this frequency from the data, the residuals shows no significant frequencies on the spectrum with the smallest FAP of 0.99 in the remaining frequencies. The linear trend observed in the radial velocities of the target star in Fig. 12 may be indicative of a long period third body, which would be detected in TTVs if there were sufficiently large number of data points spanning a longer baseline because of the large amplitude that the linear increase in RVs implies. However, a probable third body with a different set of potential physical and orbital parameters would escape detection even if it would have a shorter orbital period and the time spanned by the observational TTV data. Therefore, further spectroscopic and transit photometry observations will be needed to probe the nature of the observed TTVs in the system.

\section{CONCLUSIONS AND DISCUSSION}

\subsection{Discussion on the Parameters of HAT-P-19 b}

Within this work, we analyze a high-resolution Keck/HIRES spectrum to derive the atmospheric parameters of the host star HAT-P-19. We used the results as input parameters for the global modelling based on our high-quality transit light curves, radial velocity measurements by Hartman et al. (2011) and brightness of the star in different passbands that we collected from all-sky survey catalogues to obtain the final parameters of the planet HAT-P-19 b. We made use of the latest (second) version of EXOFAST software package (Eastman 2017, 2019) for the global modelling. Since we have an ultra-precise measurement of the stellar parallax, our SED modelling was based on the most precise distance, which enabled us to derive the stellar radius semi-empirically when the small correction applied by fitting an evolutionary track with a MESA isochrone (MIST) is also accounted for. This resulted in a more precise and accurate value for the planetary radius, which depends less on the theory of stellar evolution. Since the distance value derived from the distance modulus based on absolute and apparent magnitudes of the target in K-band ( $d=215 \pm 15$ pc) (Hartman et al. 2011) is not too different from that we derived from the Gaia parallax $\left(d \sim 200.8_{-2.6}^{+2.7} \mathrm{pc}\right)$, which is part of the reason why our final stellar and planetary parameters are similar to that published in the discovery paper by Hartman et al. (2011), the agreement is within $\pm 1 \sigma$ of the uncertainties end to end.

Based on these parameters and the definitions by Kreidberg (2018), we calculated the scale height to be $H$ $582 \mathrm{~km}$ and the amplitude of the absorption signal as $422 \pm 18$ ppm at $3.6 \mu \mathrm{m}$, and $735 \pm 28 \mathrm{ppm}$ at $4.5 \mu \mathrm{m}$, which makes it a very good candidate for James Webb Space Telescope observations to constrain its atmospheric properties, for which it is already in the prime target list (Moliere et al. 2017).

Stellar parameters of $\mathrm{M}$ and $\mathrm{K}$ dwarfs are subject to only slight changes during their main sequence lifetimes, which significantly increases the uncertainties on stellar ages derived from isochrone fitting. We found a smaller age value for HAT-P-19 as 5.1 ${ }_{-3.5}^{+4.8}$ Gyrs than found by Hartman et al. (2011) (8.8 \pm 5.2 Gyrs). The difference is very subtle. However, Mallonn et al. (2015) determined a gyrochronological age of $5.5_{-1.3}^{+1.8}$ Gyrs based on the rotation period of $P_{\text {rot }}=35.5 \pm 2.5$ days derived from the out-of-transit variation of the host star due to star spot-induced brightness changes modulated with the rotation. Our estimate for the stellar age is in agreement with their result from their photometric campaign observations within the limits of their uncertainties.

\subsection{Discussion on Transit Timing Variations}

HAT-P-19b has some key characteristics, that make it an important target for follow-up observations and further analysis. First of all, the residuals of its radial velocity data from its orbital solution that follow a linear trend may be an indicator of a gravitationally bound, yet unseen object. Such an object would cause TTVs detectable with long-baseline photometry from the ground and space. The alarming observation may be the radial velocity residuals being within a level of $60 \mathrm{~m} / \mathrm{s}$ (Hartman et al. 2011). However, we did not find a statistically significant $($ FAP $<0.05)$ periodic signal other than the seasonal observation sampling frequency in our Fourier analysis after having removed the linear trend observed in its TTV diagram due to the accumulation of uncertainties in the reference elements $\left(\mathrm{T}_{0}\right.$ and $\left.\mathrm{P}_{\text {orb }}\right)$. Nevertheless, we suggest amateur and professional observers continue their observations of its transits since a longer baseline variation is possible. Because the standard deviation of the scatter of TTV data is $\sim 2.4$ times the mean standard deviation of the observations, there may be a TTV signal with stellar or planetary origin. Some fraction of this signal can be attributed to the activity of the star, which was reported to cause a wavelength-dependent photometric variation of 3.0 to $4.7 \mathrm{mmag}$ from I to $\mathrm{V}$ band, periodi- 
cally within $35.5 \pm 2.5$ days (Mallonn et al. 2015). We did not detect such a periodicity in our Fourier analysis. However, the observed TTVs might have been affected by either the imperfect measurements of transit timings from asymmetric transit profiles due to stellar variability or imperfect weather conditions at the time, which affect only some of the light curves in our sample obtained with good sensitivity, or quasi-periodic changes in the activity level within a longer cycle. Both aspects of activity phenomenon could also have caused the observed residuals from the Keplerian fit in the radial velocity measurements. However, the amplitude of the residual RV signal $(\sim 60 \mathrm{~m} / \mathrm{s})$ can be found excessive for an activity level observed as out-of-transit photometric variations in the $\mathrm{V}$ and I bands. Although there is no correlation between these measurements and the bisector inverse span (BIS) (Hartman et al. 2011), the length of an activity cycle is incomparably longer with respect to the orbital period, and bright regions around colder stellar spots might have an opposite effect on the bisector span, negating the spot induced radial velocity variations but not affecting the brightness with a sufficient amount in the short run. All in all, we suggest follow-up observations of the transits of HAT-P-19 b to be made, and precise measurements of the mid-transit times to be used in future TTV studies, which can reveal the reasons behind the TTV signal.

\subsection{Discussion on Hot-Saturn Type Planets}

Saturn-mass planets on short-period orbits are also interesting in their own right because they are at the limit of a region in which there is a dearth of planets so-called the sub-Jovian desert on the orbital period-mass diagram (Szabó \& Kiss 2011; Beaugé \& Nesvorný 2013; Mazeh et al. 2016; Ionov et al. 2018; Owen \& Lai 2018; Nielsen et al. 2019; Szabó \& Kálmán 2019; West et al. 2019). Planets with parameters close to but above this limit at around $\mathrm{P}_{\text {orb }} \sim 2.8$ days, the number of which reached 70 at the time of this study according to NASA Exoplanet Archive ${ }^{3}$ if only the transiting planets are considered, form the boundary of the desert on the orbital period-mass plane presented here in Fig. 16. This sub-group of hot-Jupiters is often referred to as "hot-Saturns".

They are at the interface of two larger groups in terms of mass, between hot-Jupiters and Neptune-mass planets on the mass-radius diagram (Fig. 17), if $\mathrm{M}_{\mathrm{p}}=0.1 M_{\text {jup }}$ and $\mathrm{M}_{\mathrm{p}}=0.4 M_{\mathrm{jup}}$ are taken as the lower and upper mass limits of the former and latter groups, respectively. The lack of correlation between planet mass and radius has already been noticed for planets more massive than Saturn (Bakos et al. 2015), while the diversity in densities of Neptune-mass planets is obvious in Fig. 18, which was also mentioned by Bayliss et al. (2015). However, these two fundamental parameters are correlated for the hot-Saturn group of planets. In the mass-radius diagram of transiting planets less massive than $0.8 M_{\text {jup }}$ (Fig. 17), the degree of correlation changes in the transition from Neptune-mass planets after $\mathrm{M}_{\mathrm{p}} \sim 0.1 M_{\text {jup }}$ (twice the mass of the planet Neptune) and that towards hot-Jupiters at $\mathrm{M}_{\mathrm{p}} \sim 0.4 M_{\mathrm{jup}}$. the Pearson

3 https://exoplanetarchive.ipac.caltech.edu/ correlation coefficient between masses and radii of Neptunemass planets, defined as the planets between $0.025 M_{\text {jup }}$ and $0.1 M_{\text {jup }}$, is 0.36 , while it is 0.64 for Saturn-mass planets between $0.1 M_{\text {jup }}$ and $0.4 M_{\text {jup }}$. It then falls to 0.14 , when hot-Jupiter group over $0.4 M_{\text {jup }}$ (and below $0.8 M_{\text {jup }}$ ) is considered. We changed both limits by $0.01 M_{\text {jup }}$ towards both directions, and watched how the Pearson correlation coefficients and their products change on either side of the limits to be able to find better limits for the group. Although there were a couple of other good candidates, the lack of planets below a density of $2.0 \mathrm{~g} \mathrm{~cm}^{-3}$ on the mass-radius diagram in this mass interval, the alignment of hot-Saturns at the right edge of the sub-Jovian desert on the orbital periodmass plane (Fig. 16), and that they form a valley on the mass-density diagram (Fig. 18) also suggest that 0.1 and 0.4 $M_{\text {jup }}$ form a better pair for the limits of hot-Saturn planets.

Most of the planets in the Saturn-mass group (62 out of 70 in NASA Exoplanet Archive transiting sample) have inflated atmospheres, hence lower mean densities than that of the pure water. There are only $8 \rho>1 \mathrm{~g} \mathrm{~cm}^{-3}$, and two $\rho>2 \mathrm{~g} \mathrm{~cm}^{-3}$ cases within these mass limits in our sample of transiting planets. In contrast, there are $10 \rho>2 \mathrm{~g} \mathrm{~cm}^{-3}$ and only four Neptune-mass planets less dense than water between 0.06 and $0.10 M_{\text {jup }}$. Nevertheless, it should be noted that planets with larger radii are easier to detect in transit favouring less dense Saturn-mass planets to be found more easily.

Although there is a small denser sub-group, Saturnmass planets form a valley in between larger-mass hotJupiters and smaller-mass Neptunes on the mass-density plane (Fig. 18). Hot-Jupiters forming the upper boundary of the sub-Jovian desert are thought to form beyond the snow-line, then migrate inward after a sudden excitation of eccentricity due to encounters with other planets or stellar bodies, which is followed by tidal circularization stabilizing the orbit at relatively shorter periods (Matsakos \& Königl 2016; Owen \& Lai 2018; Szabó \& Kálmán 2019). The planetary migration was also suggested to be halted by a sudden loss of density within the accretion disc, mass loss due to Roche lobe overflow (Kurokawa \& Nakamoto 2014) or tidal thermalisation events (Vick et al. 2019). The outer volatile layers of these more massive planets are highly inflated because of the strong irradiation (Mordasini et al. 2015; Lundkvist et al. 2016; Demangeon et al. 2018) whereas less massive planets loose their atmospheres due to photoevaporation and end up at the bottom of the desert (Lopez \& Fortney 2014; Owen \& Lai 2018; Nielsen et al. 2019). The metallicity dependence of the photoevaporation process was highlighted by Dong et al. (2018) and Petigura et al. (2017). Mass loss can also occur due to giant planet collisions, which can strip off the atmosphere of a giant planet, leaving its core exposed as a dense Neptunian planet (Mordasini 2018).

The alignment of the shortest-period members of the hot-Saturn group on the period-mass plane forming a wall at the right edge of the sub-Jovian desert between 0.1 and $0.4 M_{\text {jup }}$ also supports this view. Then it can be argued that the planets in the Saturn-mass sub-group seem to be the least massive hot-Jupiters on shortest period orbits $\left(\mathrm{P}_{\text {orb }}>2.75\right.$ days, WASP-49b (Lendl et al. 2012) is the shortest-period planet of the group with $\mathrm{P}_{\text {orb }}=2.78$ days) that can survive the photoevaporation process if this mech- 


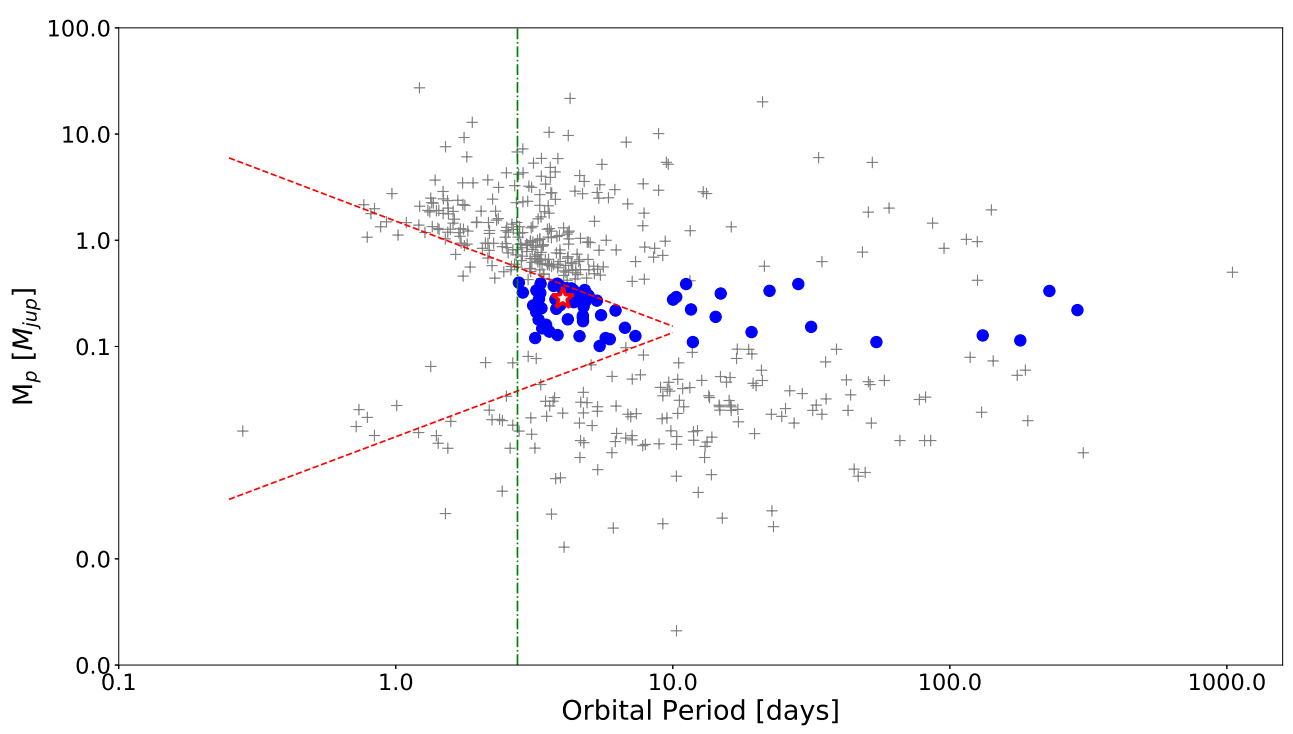

Figure 16. Orbital Period-Mass Diagram for transiting planets less massive than $0.8 M_{\text {jup }}$. Blue circles depict Saturn-mass planets as defined within this study. Gray plus marker is for all others. Red star is HAT-P-19 b, for which the error bars are smaller than the symbol. The boundaries of the sub-Jovian desert as suggested by Mazeh et al. (2016) is overplotted in red-dashed lines. Green dot-dashed line indicates the orbital period, $\mathrm{P}_{\text {orb }}=2.75$ days.

anism is assumed to be the reason behind the formation of sub-Jovian desert. Therefore, sub-Jovian desert is actually the lack of hot-Saturn planets, as defined between 0.1 and $0.4 M_{\text {jup }}$ mass limits, having orbital periods shorter than $\mathrm{P}_{\text {orb }} \sim 2.75$ days. However, Ionov et al. (2018) claimed that photoevaporation can not be the only factor for the paucity of sub-Jovians that we here described as hot-Saturns. Most recently, Szabó \& Kálmán (2019) have shown the effects of stellar parameters, namely effective temperature, metallicity, and surface gravity in the order of decreasing significance on the boundaries of the desert. The fact that most of the members of the hot-Saturn group have lower densities than both more massive hot-Jupiters and less massive hot-Neptunes (Fig. 18) also favours explanations predicting a decrease in the average density of the planet such as inflation of the outer layers due to strong stellar irradiation (Guillot \& Showman 2002; Lundkvist et al. 2016; Demangeon et al. 2018), tidal (Jackson et al. 2008) and ohmic heating (Batygin \& Stevenson 2010; Laughlin et al. 2011; Batygin et al. 2011), especially more effective for planets with smaller core-masses (Bhatti et al. 2016), and/or interaction with the accreation disc (Kurokawa \& Nakamoto 2014).

HAT-P-19 b is a typical example of the more common, less dense group of hot-Saturns closer to the upper mass and lower orbital-period limits, at the middle of the density range spanned by its fellow Saturn-mass planets with bloated atmospheres. It orbits a super-solar metallicity star $([\mathrm{Fe} / \mathrm{H}]=$ $0.243)$, which makes it an interesting example contrasting the cases like HAT-P-12 b (Hartman et al. 2009), WASP$39 \mathrm{~b}$ (Faedi et al. 2011), WASP-21 b (Bouchy et al. 2010), and numerous others in this mass regime orbiting metalpoor stars. Planetary radius (Enoch et al. 2011; Faedi et al. 2011), density (Bouchy et al. 2010; Enoch et al. 2011), and core-mass (Burrows et al. 2007; Hartman et al. 2011) have been suggested to be correlated with the host-star metallicity for Saturn-mass planets in the past. HAT-P-19b had been found to be a contradicting example (Hartman et al. 2011) to these correlations, which were, however, based only on a limited sample of Saturn-mass planets known at the time. Equipped with 70 confirmed transiting Saturn-mass planets, we needed to reconstruct these plots (Figure-11 in Hartman et al. (2011) and Figure-6 in Enoch et al. (2011)) because they had been based on only a few planets known at the time. We updated these plots with the parameters of 70 transiting-planets, for which the masses and radii are known to a few percent precision. We made use of the NASA Exoplanet Archive by limiting it in the same way as the works suggesting the correlations, computed the coremasses by interpolation in the tables given by Fortney et al. (2007) based on the parameters of the planets (masses, radii, and orbital periods) as collected from the NASA Exoplanet archive, and by using the gpc computer code (Giant Planets Cores) developed by Nestor Espinoza ${ }^{4}$, which assumes a core composition of $50 \%$ rock and $50 \%$ ice. We took the iron abundance measurements with respect to the solar-value from the most recent version of the SweetCat catalogue ${ }^{5}$ (Santos et al. 2013) as a proxy of the host-star metallicity in order to have a homogenous set. In the end, we were not able to find correlations between either the derived core-masses, or the radii with the host-star metallicities. We then further narrowed down our sample with planets having radii between 0.5 and $1.1 R_{\text {jup }}$, which limited our sample size to 46 planets, because Fortney et al. (2007) models give reli-

\footnotetext{
4 https://github.com/nespinoza/gpc

5 https://www.astro.up.pt/resources/sweet-cat/
} 


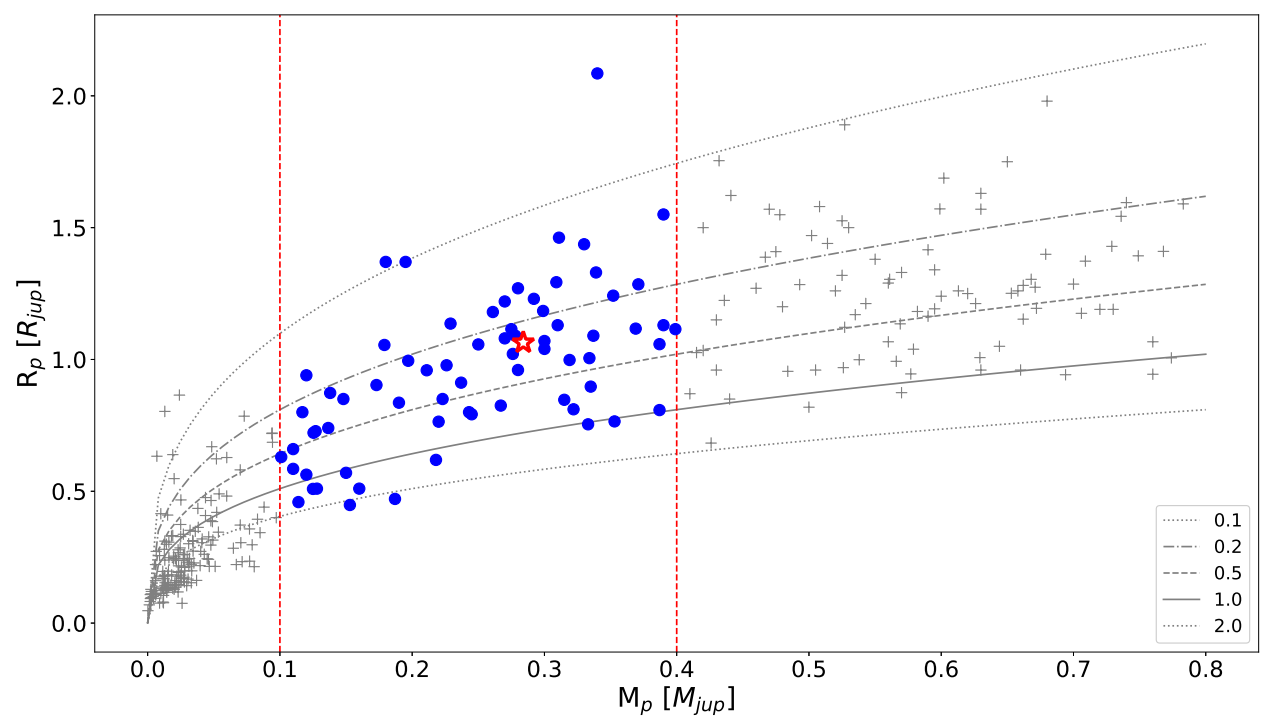

Figure 17. Mass-Radius Diagram for transiting planets less massive than $0.8 \mathrm{M}_{\mathrm{jup}}$. Symbols are the same as in Fig. 16. Vertical dashed lines limit the region where Saturn-mass planets lie. The correlation between mass and radius changes significantly before and after these limits (from $p=0.36$ to $p=0.64$ after $0.1 M_{\text {jup }}$ and down to $p=0.14$ after $0.4 M_{\text {jup }}$. A few density profiles have also been plotted, and their values are given in the legends in $\mathrm{g} / \mathrm{cm}^{3}$ units.

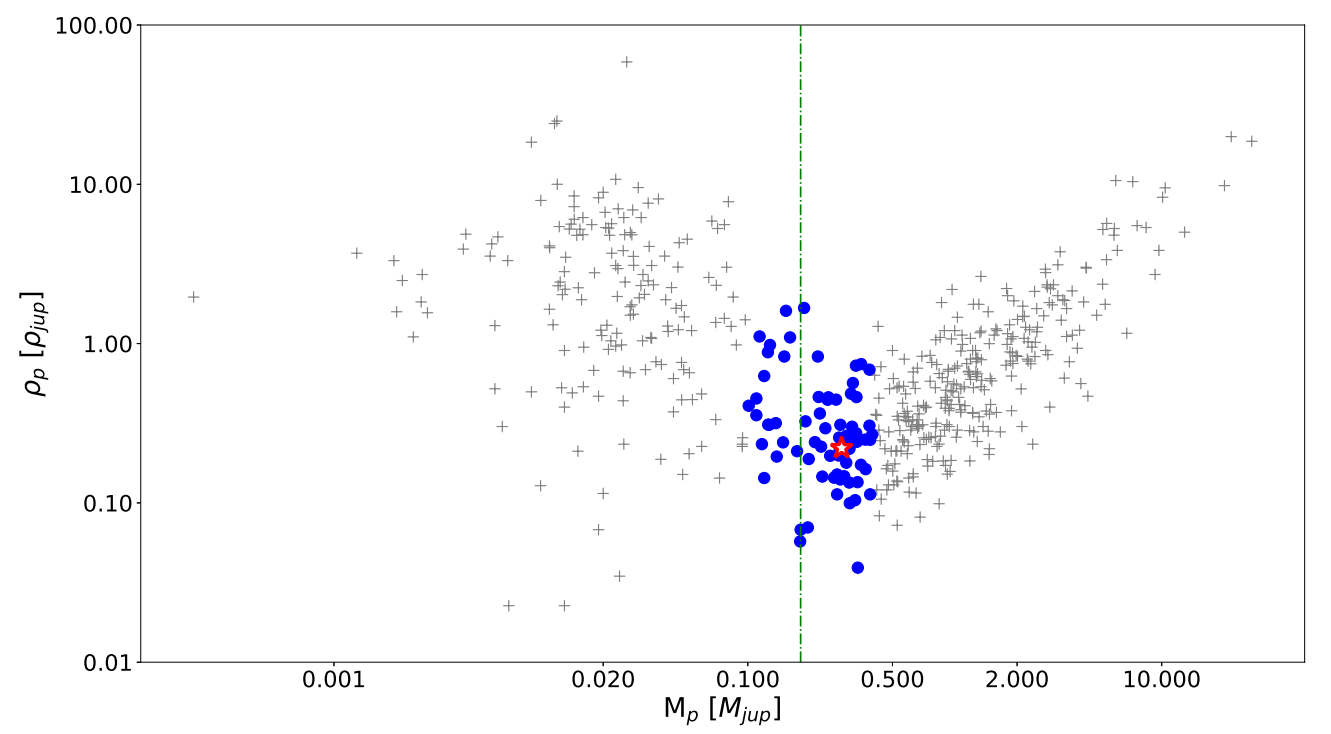

Figure 18. Mass-Density distribution for transiting planets less massive than $0.8 \mathrm{M}_{\text {jup }}$. Symbols are the same as in Fig. 18 .

able core-masses only in this radius regime. We constructed the plot combining the relations given by Hartman et al. (2011) and Enoch et al. (2011), with the mass limits for hotSaturns as defined in this study, which we present in Fig. 19. Although the core-mass and the planet radius are not correlated with the stellar metallicity, density is, as expected to be. It is apparent from the figure that higher the stellar metallicity, higher the core mass, and higher the density with a few exceptions. An interesting feature on the diagram relating the planetary properties to the stellar metallicity is the lack of dense planets orbiting metal-poor stars in the hotSaturn group with the only exception of Kepler-16 (AB) b (Doyle et al. 2011), which is a circumbinary planet. Nevertheless, we are limited to only 10 planets orbiting metal-poor stars in our sample of hot-Saturns. Potential detections of such planets around metal-poor stars within TESS mission can help us understand if the distribution of planet densities in both sides of the solar iron abundance are similar or not. 


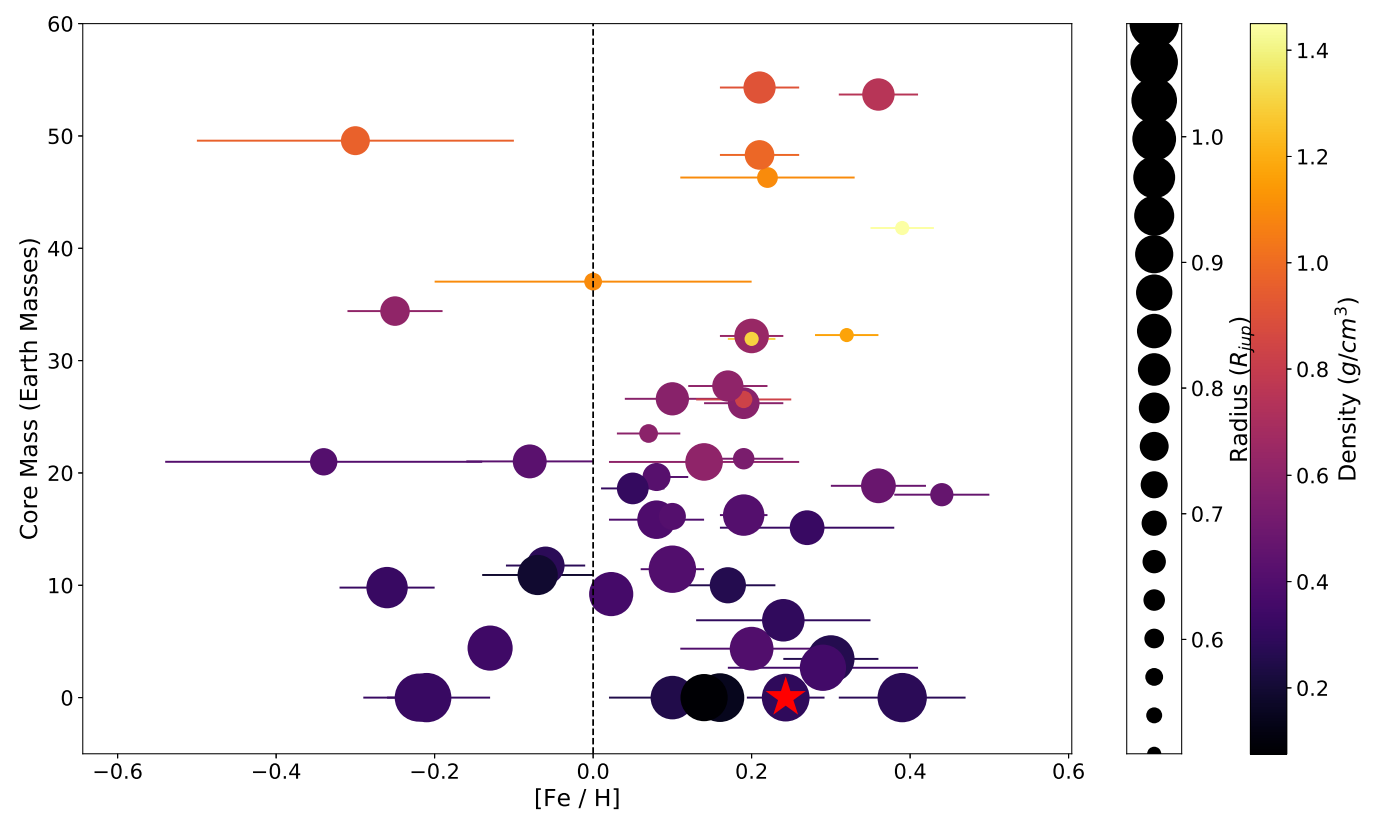

Figure 19. Host star-metallicity vs. planet core-mass diagram for transiting planets less massive than $0.8 \mathrm{M}_{\text {jup }}$ and with radii between 0.5 and $1.1 \mathrm{R}_{\text {jup }}$. Red star symbol is for HAT-P-19 b. The planet with the most massive core orbiting a metal-poor star on the upper left corner of the figure is Kepler-16 (AB) b.

On the other hand, we found, for HAT-P-19 b, a negative core-mass $\left(M_{\text {core }}=-3.14 M_{\oplus}\right)$ for 4.5 Gyr-models of Fortney et al. (2007), suggesting a core-free structure basically, and only $\mathrm{M}_{\text {core }}=7.67 M_{\oplus}$ for 1 Gyr-models, which is less than $1 \%$ of the total mass of the planet. Considering the age of the planet, updated within this study as $5.1_{-3.5}^{+4.8}$ Gyrs, it can be suggested that HAT-P-19 b has a small core. However, HAT-P-19 is a super-solar metallicity star with $[\mathrm{Fe} / \mathrm{H}]=0.24 \pm 0.05$. Planets orbiting higher metallicity stars have been found to populate the lower boundary of the sub-Jovian desert (Dong et al. 2018; Petigura et al. 2017). Hence, it was argued that the planets that appear closer to the period boundary should have larger cores (Owen \& Lai 2018; Szabó \& Kálmán 2019), for which HAT-P-19 b and all other inflated hot-Saturns are contradictory examples. In addition, it is also not in line with the argument that the metal content of the proto-planetary environment would lead to a larger core and a smaller radius (Fortney et al. 2007; Spiegel et al. 2014) if it was formed where it is observed now. Therefore, it can be suggested that the insitu formation seems unlikely for this group of planets, although it was found to be plausible for hot-Jupiters and hot-Saturns lately, which should be limited to the rare cases when a sufficiently high-mass core $\left(\sim 15 M_{\oplus}\right)$ triggers runaway gas-accretion (Batygin et al. 2016; Bailey \& Batygin 2018). If HAT-P-19 b and other Saturn-mass planets orbiting metal-rich stars had formed in close-vicinity of their hoststars, they could have been expected to have larger-cores and smaller radii (Guillot \& Showman 2002; Hartman et al. 2009; Bouchy et al. 2010; Faedi et al. 2011), which is not the case the properties of the observed group suggest.

Owen \& Lai (2018) and Giacalone et al. (2017) argued that high-eccentricity migration (Rasio \& Ford 1996; Fabrycky \& Tremaine 2007) is the most plausible migration mechanism for the planets in the upper mass boundary of the sub-Jovian desert, where the low-density hot-Saturn type planets like HAT-P-19 b lie. Massive planets $\left(\mathrm{M}_{\mathrm{p}}>0.2 M_{\text {jup }}\right)$ were suggested to survive closer to their stars although the outer layers of their atmospheres are inflated significantly due to the stellar flux and ohmic heating (Laughlin et al. 2011), they still can keep their H/He dominating bulk composition (Bayliss et al. 2015; Owen \& Lai 2018). Nevertheless, relatively smaller projected rotation-rate of HAT-P-19 in the line of sight $(v \sin i \sim 0.88 \mathrm{~km} / \mathrm{s})$ is in contradiction with the angular momentum transfer expected from the planet to the star during a high-eccentricity migration, leading to a larger rotation rate expected from the age of the star. In such cases (e.g. WASP-33 b: Collier Cameron et al. (2010), WASP-52 b: Mancini et al. (2017)) the gyrochronological ages are found to be smaller due to the larger observed rotation rates (Demangeon et al. 2018) than the ages found from isochrones. Therefore, a disk-driven migration (Lin et al. 1996; Ward 1997; Beaugé \& Nesvorný 2013) can not be ruled out since the projected rotation rate of HAT-P-19 is in agreement with a 5.1 Gyr-old star, whose gyrochronological age was found to be $\sim 5.5_{-1.3}^{+1.8}$ Gyrs by Mallonn et al. (2015). High-eccentricity migration, on the other hand, is the dominating migration mechanism in forming the upper boundary of the sub-Jovian desert as predicted by Owen \& Lai (2018), which agrees with the large discrepancies between the gyrochronological ages and that determined from isochrones in majority of the cases (Maxted et al. 2016; Demangeon et al. 2018). That said, radii of K-type stars, like HAT-P-19 itself, are underestimated by standard stellar models (Popper et al. 1997). Therefore, the discrepancy between the gyrochronological and isochrone ages is more enhanced for $\mathrm{K}$-stars (Maxted et al. 2016). Magnetic activity and a presence of 
a close-in giant planet (Hébrard et al. 2013) might be potential causes of this discrepancy other than the migration mechanisms. Since we did not find a discrepancy of the star's age we found from isochrone fitting and that found by Mallonn et al. (2015) from gyrochronology, and a strong magnetic activity indicator other than a few percent out-oftransit light curve modulation; HAT-P-19 b might have been migrated to its location via disk-driven migration. In brief, whatever the valid migration mechanism is for HAT-P-19 b, it turned out to be massive enough not to loose its atmosphere due to photoevaporation and/or far enough from its star not to get tidally disrupted.

\section{ACKNOWLEDGEMENTS}

We gratefully acknowledge the support by The Scientific and Technological Research Council of Turkey (TÜBİTAK) with the project $116 \mathrm{~F} 350$. We thank TÜBİTAK for the partial support in using T100 telescope with the project numbers 12CT100-378 and 16CT100-1096. TD acknowledges support from MIT's Kavli Institute as a Kavli postdoctoral fellow. This research has made use of the SVO Filter Profile Service (http://svo2.cab.inta-csic.es/theory/fps/) supported by the Spanish MINECO through grant AYA2017-84089. Some of the data presented herein were obtained at the W. M. Keck Observatory, which is operated as a scientific partnership among the California Institute of Technology, the University of California and the National Aeronautics and Space Administration. The Observatory was made possible by the generous financial support of the W. M. Keck Foundation. We thank all the amateur and professional observers who report their data to Exoplanet Transit Database (ETD), permitted us to use them, and answered all of our never-ending questions. We acknowledge the work put on the computer code gpc by Nestor Espinoza. We thank Heather Knutson for kindly sharing the Spitzer light curves of the target with us. We also thank Jason Eastman for his guidance and help by answering our questions in using the EXOFAST software pakcage.

\section{DATA AVAILABILITY}

The spectroscopic observations underlying this article are available in Keck Observatory Archive at https://www2.keck.hawaii.edu/koa/public/koa.php. TESS and Spitzer light curves were derived from sources in the public domain of Barbara A. Mikulski Archive for Space Telescopes at https://mast.stsci.edu/. Passband brightness data were collected from different sources in literature (see Table 3 for details). FLWO light curves are from Hartman et al. (2011). Some of the light curves to derive mid-transit times were downloaded from Exoplanet Transit Database at http://var2.astro.cz/ETD/. All other light curves appearing for the first time in this article, and the derived mid-transit times will be shared on reasonable request to the corresponding author. Parameters of transiting planets used in the study were gathered from the NASA Exoplanet Archive.

\section{REFERENCES}

Alam, S., et al., 2015, ApJS, 219, 12

Asplund, M., et al., 2009, ARA\&A, 47, 1

Astropy Collaboration, 2013, A\&A, 558, A33

Astropy Collaboration, 2018, AJ, 156, 123

Bailey, E., Batygin, K., ApJ, 866, 2

Baştürk Ö., et al., 2015, ASPC, 496, 370

Bakos, G.Á. et al., 2015, ApJ, 813, 111

Bayiss, D., et al., 2015, AJ, 150, 49

Batygin, K., Stevenson, D.J., 2010, ApJ, 714, L238

Batygin, K., Stevenson, D.J., Bodenheimer, P.H., 2011, ApJ, 738,

$$
1
$$

Batygin, K., et al., 2016, ApJ, 829, 114

Beaugé, C., Nesvorný, D., 2013, ApJ, 763, 12

Bhatti, W., 2016, arXiv:1607.00322

Bonomo, A.S., 2017, A\&A, 602, 107

Bouchy, F., 2010, A\&A, 519, A98

Brewer, J, et al., 2016, ApJS, 225, 32

Burrows, A., Hubeny, I., Budaj, J., Hubbard, W.B., 2007, ApJ, 661,502

Castelli, F., Kurucz, R.L., 2004, PASP, 2003., 20

Chambers, K.C., et al., 2016, Vizier Online Data Catalog: PANSTARRS1 Surveys, yCat, 2349

Claret, A., Bloemen, S. 2011, A\&A, 529, A75

Claret, A., Bloemen, S. 2011, A\&A, 529, A75

Collins, et al., 2017, AJ, 153, 77

Blanco-Cuaresma, S., et al., 2014, A\&A, 569, 111

Blanco-Cuaresma, S., 2019, MNRAS, 486, 2075

Cubillos, P.E., et al., 2017, ApJ, 849, 145

Cutri R. M., et al., 2003, VizieR Online Data Catalog: 2MASS All-Sky Catalog of Point Sources, yCat, 2246

Cutri R. M., et al., 2014, VizieR Online Data Catalog: AllWISE Data Release, yCat, 2328

Demangeon, O.D.S., et al., A\&A, 610, A63

Deming, D., et al. , 2015, ApJ, 805, 132

Dong, S., et al., PNAS, 115, 266

Doyle, L.R., et al., Science, Vol. 333, Issue 6049, 1602

Enoch, B., et al., 2011, MNRAS, 410, 1631

Eastman J., 2017, ascl:1710.003

Eastman J., 2019, arXiv:1907.09480

Fabrycky, D., Tremaine, S. 2007, ApJ, 669, 1298

Faedi, F., et al., 2011, A\&A, 531, A40

Feltzing, S., Gonzalez, G. 2001, A\&A, 367, 253

Fortney, J.J., Marley, M.S., 2007, ApJ, 659, 1661

Fuhrmann, K. 2004, AN, 325, 3

Fulton B. J., et al., 2011, AJ, 142, 84

Gaia Collaboration, 2016, A\&A, 595, A1

Gaia Collaboration, et al., 2018, A\&A, 616, A1

Giacalone, S., et al., 2017, AJ, 154, 192

Gilmore, G., et al., 2012, The Messenger, 147, 25

Gray, D. F. 1994, PASP, 106, 1248

Guillot, T., Showman, A. P, 2002, A\&A, 385, 156

Hartman J. D., et al., 2009, ApJ, 706, 785H

Hartman J. D., et al., 2011, AJ, 726, 52

Hébrard G., et al., 2013, A\&A, 549, A134

Henden A. A., et al., 2016, VizieR Online Data Catalog: AAVSO Photometric All Sky Survey (APASS) DR9, yCat, 2326

Honeycutt, R. K., 1992, PASP, 104, 435

Ionov, D.E., Pavlyuchenkov, Y.N., Shematovich, V.I., MNRAS, 476, 5639

Jackson, B., Barnes, R., Greenberg, R. 2008, MNRAS, 391, 237

Kammer, J.A., et al. 2015, ApJ, 810, 118

Kreidberg, L., 2018, Handbook of Exoplanets, Exoplanet Atmosphere Measurements from Transmission Spectroscopy and Other Planet Star Combined Light Observations. In: Deeg H., Belmonte J. (eds) Handbook of Exoplanets. Springer, Cham

Kupka, F. G., et al., 2000, Baltic Astronomy, 9, 590 
Kurokawa, H.; Nakamoto, T., 2014, ApJ, 783, 54

Kurucz R. L., Avrett E. H., 1981. SAO Special Report, 391

Kurucz R. , 1993, ATLAS9 Stellar Atmosphere Programs and $2 \mathrm{~km} / \mathrm{s}$ grid. Kurucz CD-ROM No.13. Cambridge, Mass.: Smithsonian Astrophysical Observatory, p. 1993 . 13

Laughlin, G., Crismani, M., Adams, F. C., 2011, ApJ, 729, L7

Lendl M., et al., 2012, A\&A, 544, A72

Lendl M., et al., 2016, A\&A, 587, A67

Lendl M., et al., 2019, MNRAS, 482, 301

Lin, D. N. C., Bodenheimer, P., Richardson, D. C. 1996, Nature, 380,606

Lopez, E.D., Fortney, J.J., ApJ, 792, 1

Lundkvist, M. S., et al., Nature Communications, 7, 11201

Maciejewski G., et al., 2018, IBVS, 6243

Mallonn M., et al., 2015, A\&A, 580, A60

Mancini L., et al., 2014, MNRAS, 443, 2391

Mancini L., et al., 2017, MNRAS, 465, 843

Matsakos, T., Königl, A., 2016, ApJ, 820, L8

Maxted, P. F. L., Serenelli, A. M., Southworth, J., A\&A, 577, A90

Mills, S. M., Fabricky, D., ApJL, 838, L11

Mazeh T., Holczer T., Faigler S., 2016, A\&A, 589, A75

Mordasini C., et al., 2015, International Journal of Astrobiology., 14,201

Mordasini C., 2018, Handbook of Exoplanets, ISBN 978-3319-55332-0. Springer International Publishing AG, part of Springer Nature, 2018, id.143

Moliere, P., et al., 2017, A\&A, 600, 10

Nielsen, L. D., et al., MNRAS, 489, 2478

Nikolov, N., et al., Nature, 557, 526

Paxton, B., et al., 2011, ApJS, 192, 3

Paxton, B., et al., 2013, ApJS, 208, 4

Paxton, B., et al., 2015, ApJS, 220, 15

Pearson, K.A., et al., 2019, AJ, 157, 21

Piskunov, N. E., et al., 1995, A\&ASS, 112, 525

Popper D. M., 1997, AJ, 114, 1195

Rampalli, R., 2019, AJ, 158, 62

Rasio, F. A., Ford, E. B. 1996, Science, 274, 954

Rodrigo, C., Solano, E., Bayo, A. 2012, http://ivoa.net/documents/Notes/SVOFPS/index.html

Rodrigo, C., Solano, 2013, http://ivoa.net/documents/Notes/SVOFPSDAL/index.html

Ryabchikova, T., et al., 2015, Physica Scripta, 90, 054005

Santos, N. C., Israelian, G., Mayor, M., 2004, A\&A, 415, 1153

Seeliger M., et al., 2015, MNRAS, 451, 4060

Owen, J.E., Lai, D., 2018, MNRAS, 479, 5012

Petigura, E.A. et al., 2017, AJ, 153, 142

Ricker, G.R. et al., 2015, JATIS, vol 1, id. 014003

Santos, N., et al., 2013, A\&A, 556, A150

Schlegel, D.J., Finkbeiner, D.P., Davis, M., 1998, AJ, 500, 525

Sing, D.K., et al., 2011, A\&A, 527, 73

Southworth J., et al., 2009, MNRAS, 396, 1023

Southworth J., et al., 2014, MNRAS, 444, 776

Spiegel D.S., et al., 2016, Proceedings of the National Academy of Sciences, Volume 111, Issue 35, 2014, pp.12622-12627

Stassun, K. G., Torres, G., 2018, AJ, 862, 61

Stassun, K. G., et al., 2019, AJ, 158, 138

Szabó, Gy.M., Kálmán, Sz., MNRAS, 485, L116

Szabo G. M., Kiss L. L., 2011, ApJ, 727, L44

Torres, G., Andersen, J., and Giménez, A., 2010, A\&ARv, 18, 67

Tsantaki, M., et al., 2013, A\&A, 555, A150

Teske, J. K., et al., 2015, ApJ, 801, 10

Valenti, J. A., Fischer, D. A. 2005, ApJS, 159, 141

Vick, M., Lai, D., Anderson, K.R., 2019, MNRAS, 484, 5645

Ward, W. R., 1997, Icarus, 126, 261

West, R. G., et al., 2019, MNRAS, 486, 5094

Winn J. N., et al., 2008, ApJ, 683, 1076

Wyttenbach A., et al., 2017, A\&A, 602, 36
Zacharias. N., et al., 2005, VizieR Online Data Catalog: NOMAD Catalog, yCat, 1297

This paper has been typeset from a $\mathrm{T}_{\mathrm{E}} \mathrm{X} / \mathrm{LAT}_{\mathrm{E}} \mathrm{X}$ file prepared by the author. 